\title{
Supporting information: Dynamic Properties of the Photosensory Domain of Deinococcus Radiodurans Bacteriophytochrome
}

\author{
Giovanni Battocchio ${ }^{1}$, Ronald González ${ }^{1}$, Aditya G. Rao ${ }^{2}$, Igor \\ Schapiro $^{2}$, and Maria Andrea Mroginski ${ }^{1}$ \\ ${ }^{1}$ Institut für Chemie, Technische Universität Berlin, Sekr. PC 14, \\ Strasse des 17. Juni 135, D-10623 Berlin \\ ${ }^{2}$ Fritz Haber Center for Molecular Dynamics Research Institute of \\ Chemistry, The Hebrew University of Jerusalem Jerusalem, 91904, \\ Israel
}

\section{Computational Details}

All simulations were performed on CUDA-enabled graphics processing unit (GPU). Both NAMD(v2.12) and AMBER(v16) software benefit from the use of CUDA acceleration, with AMBER(v16) being more efficient than NAMD(v2.12) (see table S1), making AMBER the preferable choice for future long calculations.

Table S1: Comparison of software speed on a single NVIDIA RTX $2080 \mathrm{Ti}$.

\begin{tabular}{|l|l|l|l|}
\hline Software & \# CPU & t $\forall$ ns (h) & ns/day \\
\hline NAMD(v2.12) & 6 & 5,63 & 4,3 \\
\hline AMBER(v16) & 1 & 0,31 & 77,2 \\
\hline
\end{tabular}

\section{New torsional parameters BV chromophore}

In this work, we carried out the same strategy proposed in the Ref. [1]. for generating the torsional parameters of the Biliverdin (BV) chromophore. The quantum mechanics $(\mathrm{QM})$ and molecular mechanics $(\mathrm{MM})$ potential energy surfaces (Figure S2.) were scanned in steps of $5^{\circ}$ by means of constrains energy minimizations. In each step, just the dihedral angle is kept fixed and the rest of the molecule is allowed to relax.

As we can see in figure $\mathrm{S} 1$, the new torsional parameters adequately describe the rotation around the $\mathrm{C}=\mathrm{C}$ between rings $\mathrm{C}$ and $\mathrm{D}$ in the $\mathrm{BV}$-chromophore. The $\mathrm{QM}$ potential energy surface presents a local minimum $\left(\sim 17^{\circ}\right)$ and a global minimum $\left(\sim 180^{\circ}\right)$; the same behavior is observed with the MM potential energy surface using 
the new torsional parameters. A good description of the rotation between rings $\mathrm{C}$ and $\mathrm{D}$ is crucial for studying conformational changes by using molecular dynamics (MD) simulations.

Table S2: Optimized Parameters for Dihedral Angle of the BV Chromophore.

\begin{tabular}{|l|l|l|l|}
\hline Dihedral parameter & $K_{\chi}(\mathrm{Kcal} / \mathrm{mol})$ & $\mathrm{n}$ & $\chi(\mathrm{deg})$ \\
\hline CPA-CPY2-CA-CPY5 & 1.70 & 4 & 0.0 \\
\hline CPA-CPY2-CA-CPY5 & 0.10 & 3 & 0.0 \\
\hline CPA-CPY2-CA-CPY5 & 9.00 & 2 & 180.0 \\
\hline CPA-CPY2-CA-CPY5 & 0.10 & 1 & 0.0 \\
\hline
\end{tabular}

Table S3: Optimized Parameters for Improper Angle of the BV Chromophore.

\begin{tabular}{|l|l|l|l|}
\hline Improper parameter & $K_{\psi}\left(\mathrm{Kcal} / \mathrm{mol} / \mathrm{rad}^{2}\right)$ & $\varnothing$ & $\psi_{0}(\mathrm{deg})$ \\
\hline CA-CPY1-NR1-CPY3 & 140.0000 & 0 & 0.0000 \\
\hline CA-CPY5-NR1-CPY2 & 7.0000 & 0 & 0.0000 \\
\hline
\end{tabular}

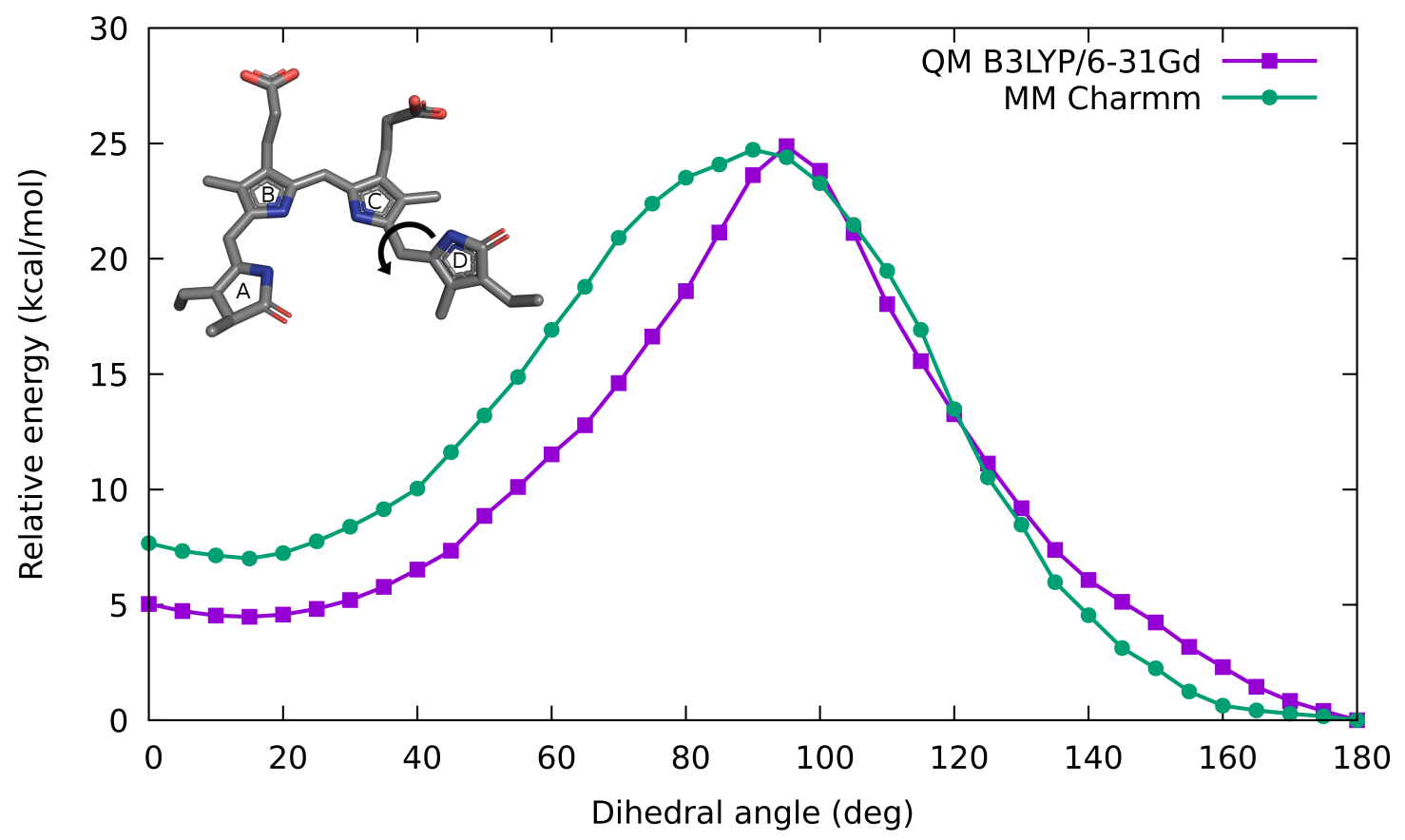

Figure S1: Potential energy surfaces for the dihedral angle between rings $\mathrm{C}$ and $\mathrm{D}$ of BV chromophore. The purple curve corresponds to the target data derived using quantum chemical calculations (DFT/B3LYP/6-31Gd) and the green curve denote the potential energy surface (MM) using the torsional parameters of the Table S1. The fitting was performed with the python program "fit_dihedral.py" (Ref. [2]) and the perl script "fit_dihedral_parameters_paper.plx" (Ref.[3]).

\section{AMBER parameters for the BV chromophore}

For the simulations with AMBER ff14SB [4] protein force field the parameters for the BV chromophore were derived on the basis of the phycocyanobilin (PCB) chro- 
mophore. For this purpose we used the mdgx tool [5, 6] of the AMBER(v16) program suite. Due to the presence of two extra double bonds in BV compared to PCB, two new atom types ( $\mathrm{m} 5$ and $\mathrm{m} 6$ ) were introduced to decouple torsional parameters having the same atom types. A list of atoms, their corresponding atom types and partial charges are given in Table S4. The partial charges for the full chromophore were derived at $\mathrm{HF} / 6-31 \mathrm{G}^{*}$ level of theory using a multi-conformational RESP method, consistent with the derivation of the ff14SB parameters. To reduce the computational demand, torsional parameters surrounding the two double bonds were derived from reduced fragments of the chromophore. The level of theory employed for the derivation of dihedrals and bond angles was MP2/cc-pVTZ.

To validate the torsional parameters surrounding the double bonds, MM-minimized conformations of the reduced fragments were generated by systematically increasing the torsions from $-180^{\circ}$ to $+180^{\circ}$ in steps of $10^{\circ}$. Both dihedral profiles exhibit a single minimum. There is a close agreement between the calculated energy profiles from the parametrized force field (MM) and the MP2 profiles (QM). Since the isomerization is unlikely on the ground state, the parametrization process was focused on the dihedrals around the ground state minimum. The standard deviation (SD) between the QM and the MM profiles for the dihedral connecting the cysteine and the A-ring is $3.73 \mathrm{kcal} / \mathrm{mol}$ and that of ethyl group of D ring is $4.79 \mathrm{kcal} / \mathrm{mol}$. The angles and dihedrals parameterized are listed in Table S5 and S6.

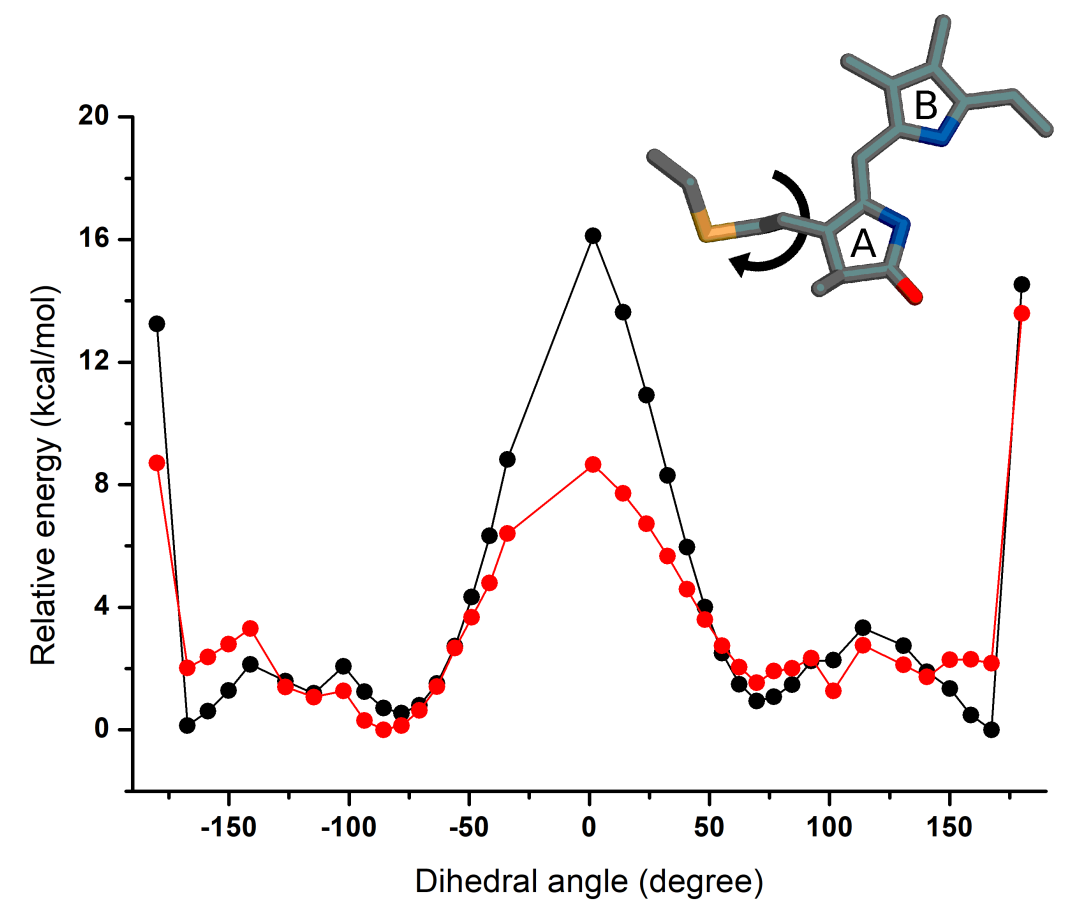

Figure S2: Comparison of QM (red) and MM (black) dihedral profiles in gas-phase. 


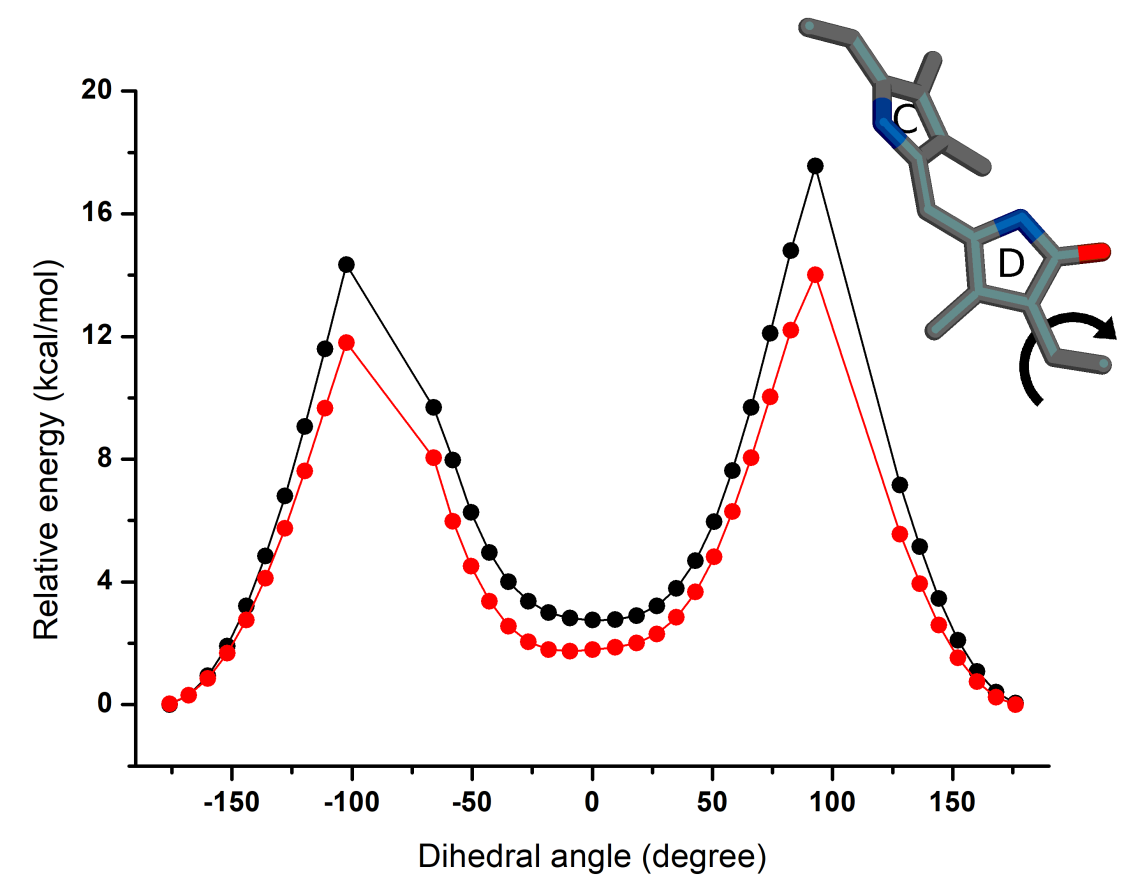

Figure S3: Comparison of QM (red) and MM (black) dihedral profiles in gas-phase.

Table S4: List of atoms, atom types and the partial charges. 


\begin{tabular}{|c|c|c|c|c|c|}
\hline Atom name & Atom type & Partial charge & Atom name & Atom type & Partial charge \\
\hline $\mathrm{N}$ & $\mathrm{N}$ & -0.4157 & HBC1 & hc & -0.0122 \\
\hline $\mathrm{H}$ & $\mathrm{H}$ & 0.2719 & HAC1 & hc & 0.09627 \\
\hline $\mathrm{CA}$ & CX & 0.06805 & CMC & c3 & -0.19087 \\
\hline $\mathrm{CB}$ & $2 \mathrm{C}$ & -0.00787 & HMC2 & hc & 0.08456 \\
\hline HB3 & H1 & 0.06777 & HMC3 & hc & 0.08456 \\
\hline HB2 & H1 & 0.06777 & HMC1 & he & 0.08456 \\
\hline SG & $\mathrm{S}$ & -0.20305 & CHD & $\mathrm{m} 3$ & -0.51088 \\
\hline $\mathrm{CBA}$ & c3 & -0.19705 & C1D & $\mathrm{m} 4$ & 0.43462 \\
\hline CAA & $\mathrm{c} 2$ & -0.01658 & $\mathrm{C} 2 \mathrm{D}$ & $\mathrm{cd}$ & -0.16089 \\
\hline $\mathrm{C} 3 \mathrm{~A}$ & $\mathrm{~m} 5$ & -0.21384 & C3D & $\mathrm{cd}$ & 0.17166 \\
\hline $\mathrm{C} 2 \mathrm{~A}$ & c3 & 0.19825 & CAD & $\mathrm{m} 6$ & -0.35323 \\
\hline C1A & $\mathrm{c}$ & 0.58741 & $\mathrm{CBD}$ & c2 & -0.24325 \\
\hline $\mathrm{N}_{A}$ & na & -0.37516 & HBD2 & ha & 0.18664 \\
\hline $\mathrm{C} 4 \mathrm{~A}$ & $\mathrm{ca}$ & 0.15102 & HBD1 & ha & 0.18664 \\
\hline CHB & $\mathrm{m} 1$ & -0.31131 & HAD1 & ha & 0.19921 \\
\hline C1B & $\mathrm{cc}$ & 0.16787 & $\mathrm{C} 4 \mathrm{D}$ & $\mathrm{c}$ & 0.67711 \\
\hline $\mathrm{N}_{B}$ & na & -0.41128 & $\mathrm{O}_{D}$ & $\mathrm{o}$ & -0.54791 \\
\hline C4B & $\mathrm{cc}$ & -0.08992 & $\mathrm{~N}_{D}$ & na & -0.70666 \\
\hline C3B & $\mathrm{cd}$ & 0.09193 & HAG & $\mathrm{hn}$ & 0.43168 \\
\hline $\mathrm{C} 2 \mathrm{~B}$ & $\mathrm{~cd}$ & 0.04079 & CMD & c3 & -0.22612 \\
\hline CMB & c3 & -0.15804 & HMD3 & hc & 0.10736 \\
\hline HMB3 & hc & 0.0692 & HMD2 & hc & 0.10736 \\
\hline HMB1 & hc & 0.0692 & HMD1 & hc & 0.10736 \\
\hline HMB2 & hc & 0.0692 & HHD1 & ha & 0.21373 \\
\hline $\mathrm{CAB}$ & c3 & -0.11566 & HAF & hn & 0.36685 \\
\hline HAB2 & hc & 0.08662 & HHC1 & ha & 0.14728 \\
\hline HAB1 & hc & 0.08662 & HAE & hn & 0.32221 \\
\hline CBB & c3 & -0.1722 & HHB1 & ha & 0.16703 \\
\hline CGB & c3 & 0.7994 & HAD & $\mathrm{hn}$ & 0.3548 \\
\hline O1B & $\mathrm{O}$ & -0.8014 & $\mathrm{O}_{A}$ & $\mathrm{O}$ & -0.59699 \\
\hline $\mathrm{O} 2 \mathrm{~B}$ & o & -0.8014 & $\mathrm{H} 2 \mathrm{~A}$ & hc & 0.0175 \\
\hline HBB1 & hc & -0.0122 & CMA & c3 & -0.19754 \\
\hline HBB2 & hc & -0.0122 & HMA3 & hc & 0.07134 \\
\hline $\mathrm{CHC}$ & $\mathrm{m} 2$ & 0.10498 & HMA2 & hc & 0.07134 \\
\hline $\mathrm{C} 1 \mathrm{C}$ & $\mathrm{cd}$ & -0.07964 & HMA1 & hc & 0.07134 \\
\hline $\mathrm{N}_{C}$ & na & -0.45348 & HAA1 & ha & 0.15374 \\
\hline $\mathrm{C} 4 \mathrm{C}$ & $\mathrm{cd}$ & 0.30552 & HBA2 & h1 & 0.14841 \\
\hline $\mathrm{C} 3 \mathrm{C}$ & $\mathrm{cc}$ & -0.09616 & HBA3 & h1 & 0.14841 \\
\hline $\mathrm{C} 2 \mathrm{C}$ & $\mathrm{cc}$ & 0.06515 & HA & H1 & 0.0766 \\
\hline CAC & c3 & -0.10601 & $\mathrm{C}$ & $\mathrm{C}$ & 0.5973 \\
\hline HAC2 & he & 0.09627 & $\mathrm{O}$ & $\mathrm{O}$ & -0.5679 \\
\hline CBC & c3 & -0.1722 & $\mathrm{O} 2 \mathrm{C}$ & $\mathrm{O}$ & -0.8014 \\
\hline CGC & c3 & 0.7994 & HBC2 & hc & $\begin{array}{l}-0.0122 \\
\end{array}$ \\
\hline $\mathrm{O} 1 \mathrm{C}$ & $\mathrm{O}$ & -0.8014 & & & \\
\hline
\end{tabular}


Table S5: Optimized parameters for angles.

\begin{tabular}{|c|c|c|}
\hline Angle parameter & Angle force constant $\left(k_{w}\right)$ & Equilibrium value $\left(\Theta_{0}\right)$ \\
\hline c3-c2-m5 & 23.0400 & 123.49 \\
\hline cd-m6mc2 & 32.1312 & 118.40 \\
\hline
\end{tabular}

Table S6: Optimized parameters for dihedrals.

\begin{tabular}{|c|c|c|c|}
\hline Dihedral parameter & Dihedral force constant $\left(k_{j}\right)$ & Phase shift $(\gamma)$ & Periodicity $(j)$ \\
\hline na-cd-m3-m4 & 5.58760 & 180.0 & 2.0 \\
\hline cc-cd-m3-m4 & 6.24350 & 180.0 & 2.0 \\
\hline 2C-S -c3-c2 & -1.53513 & 0.0 & 3.0 \\
\hline S -c3-c2-m5 & -0.27350 & 0.0 & 2.0 \\
\hline cd-cd-m6-c2 & 0.12374 & 180.0 & 2.0 \\
\hline cd-cd-m6-ha & 1.40864 & 180.0 & 2.0 \\
\hline c3-c2-m5-c3 & 8.76865 & 180.0 & 2.0 \\
\hline c3-c2-m5-ca & 8.71560 & 180.0 & 2.0 \\
\hline
\end{tabular}




\section{Final structures confronted}

a)
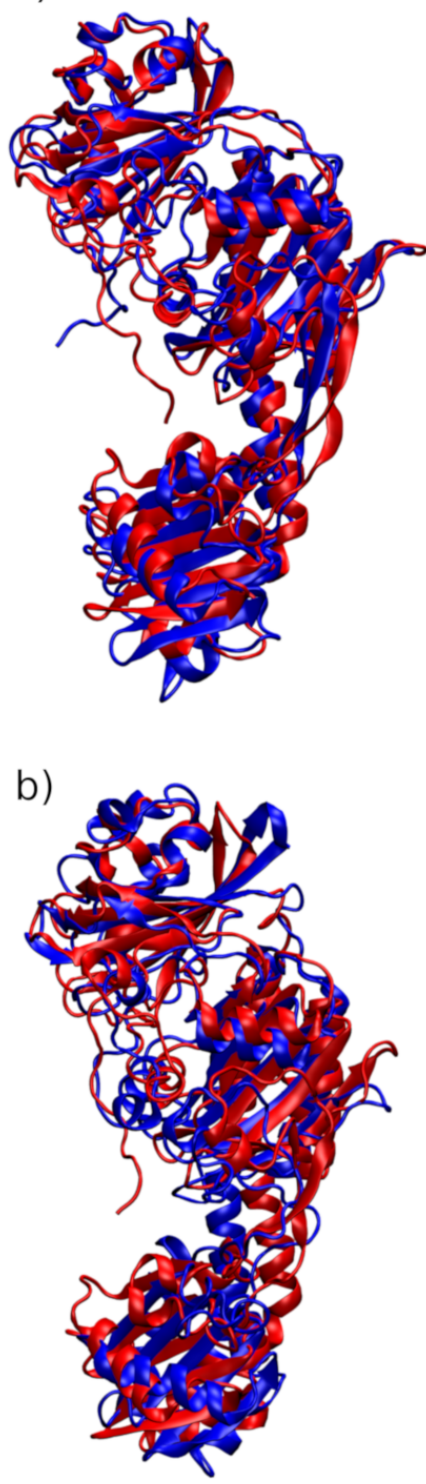
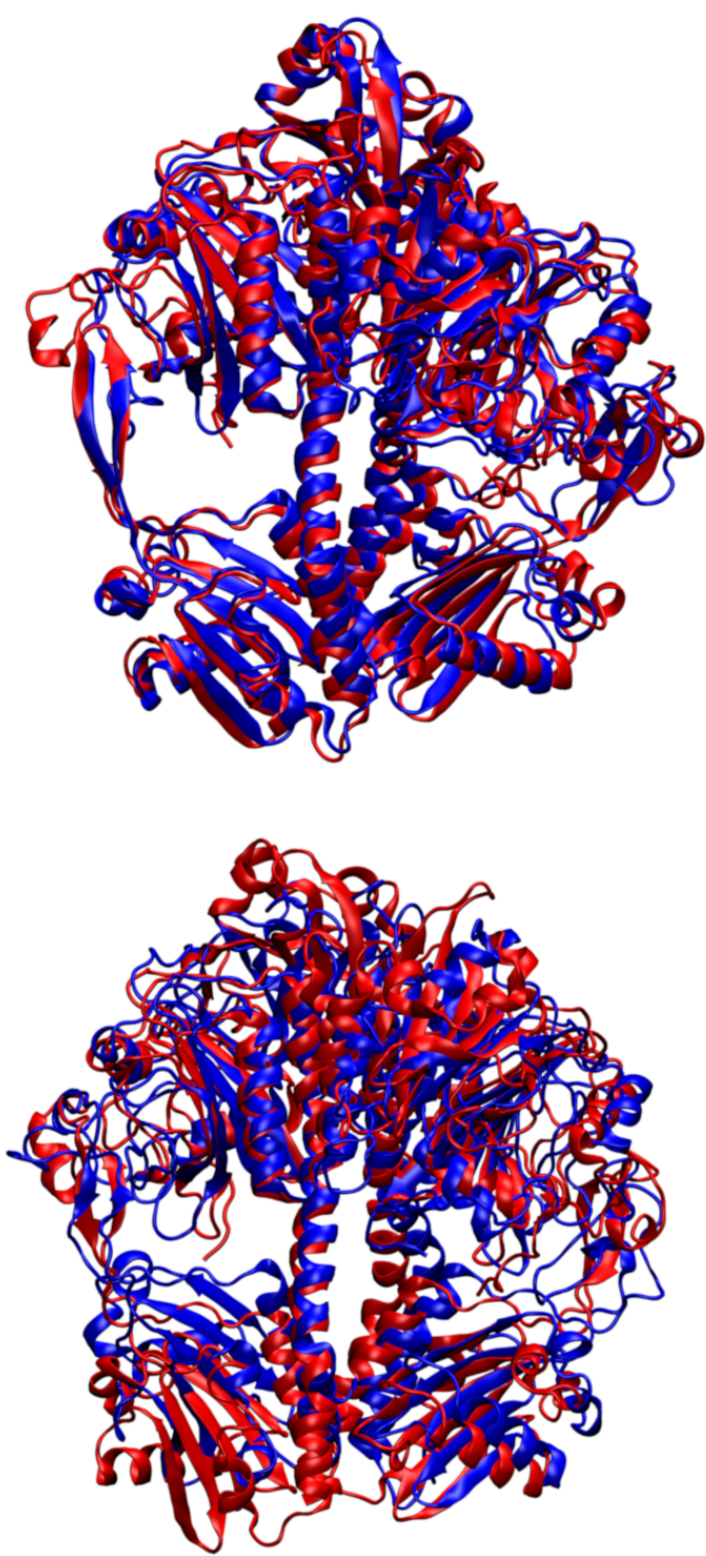

Figure S4: DrBphP structures after 500 ns MD simulations. a) CHARMM27, monomer and dimer. cMD in red, aMD in blue. b) AMBER ff14SB, monomer and dimer, cMD in red, GaMD in blue.

The structures resulting from the $500 \mathrm{~ns}$ classical and accelerated MD simulations are, for most parts of the protein, comparable. In all four cases we observe the same PHY domain movements which are described in the main text. AMBER final aMD structures, however, exhibit higher deviations with respect to the corresponding final cMD structure. The main difference is the amplitude of the kink angle of the helical spine which shows a larger value in the case of the GaMD. The reason for these structural differences is the higher effective acceleration used in AMBER GaMD compared to the one employed for the CHARMM aMD simulations, which 
leads to a more extensive sampling of the conformational space as depicted in the Ramachandran plots (main text) for the dimer. This effect is also reflected in the higher RMSD values for AMBER GaMD compared to the CHARMM aMD (see main text).

\section{RMSF}

The Root mean square fluctuation (RMSF) indicates the flexibility of a molecule in general, is defined as

$$
R M S F=\sqrt{\frac{1}{T} \sum_{t=1}^{T}\left(r_{i, t}-\left\langle r_{i}\right\rangle\right)^{2}}
$$

i.e. the deviation of the atom $i$ from his mean position at time-step t. We again used the $\mathrm{C}_{\alpha}$-backbone structure as reference.

In general with the rmsd can give more information of changes related to a particular conformation, while the rmsf indicate when the overall flexibility is increased.

Table S7: Mean RMSF values for the $\mathrm{C}_{\alpha}$-backbone, after aligning with respect to the GAF domain, during 500 ns simulation, cMD. Units are $\AA$.

\begin{tabular}{|c|c|c|}
\hline $\mathrm{C}_{\alpha}$-RMSF & Monomer & Dimer \\
\hline CHARMM27 & 2.073 & 2.166 \\
\hline AMBER ff14SB & 1.953 & 1.653 \\
\hline
\end{tabular}

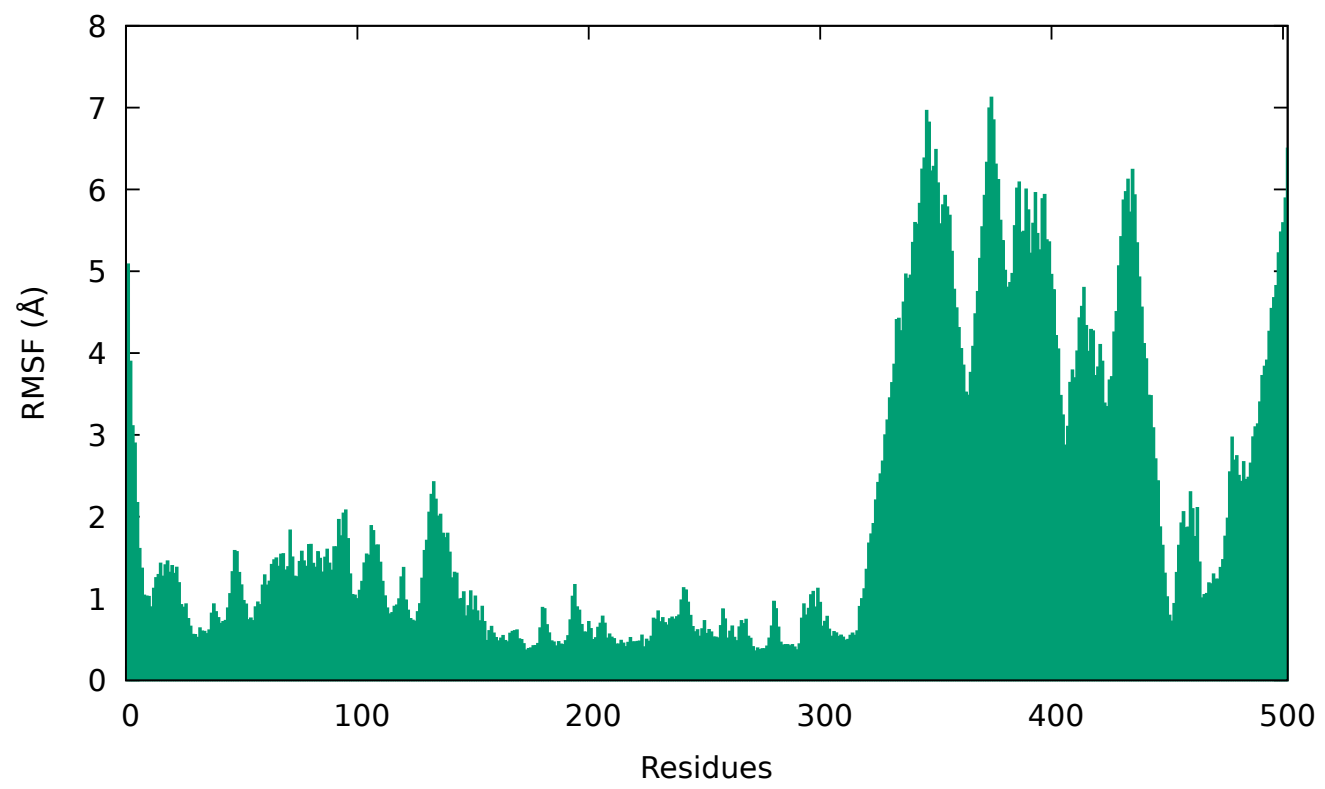

Figure S5: Mean RMSF of $\mathrm{C}_{\alpha}$-backbone atoms of each residue of $\mathrm{DrBPhP}$ monomer computed during the last 400 ns cMD simulation using CHARMM27 after alignment $\mathrm{w} / \mathrm{r} / \mathrm{t}$ the GAF domain. 


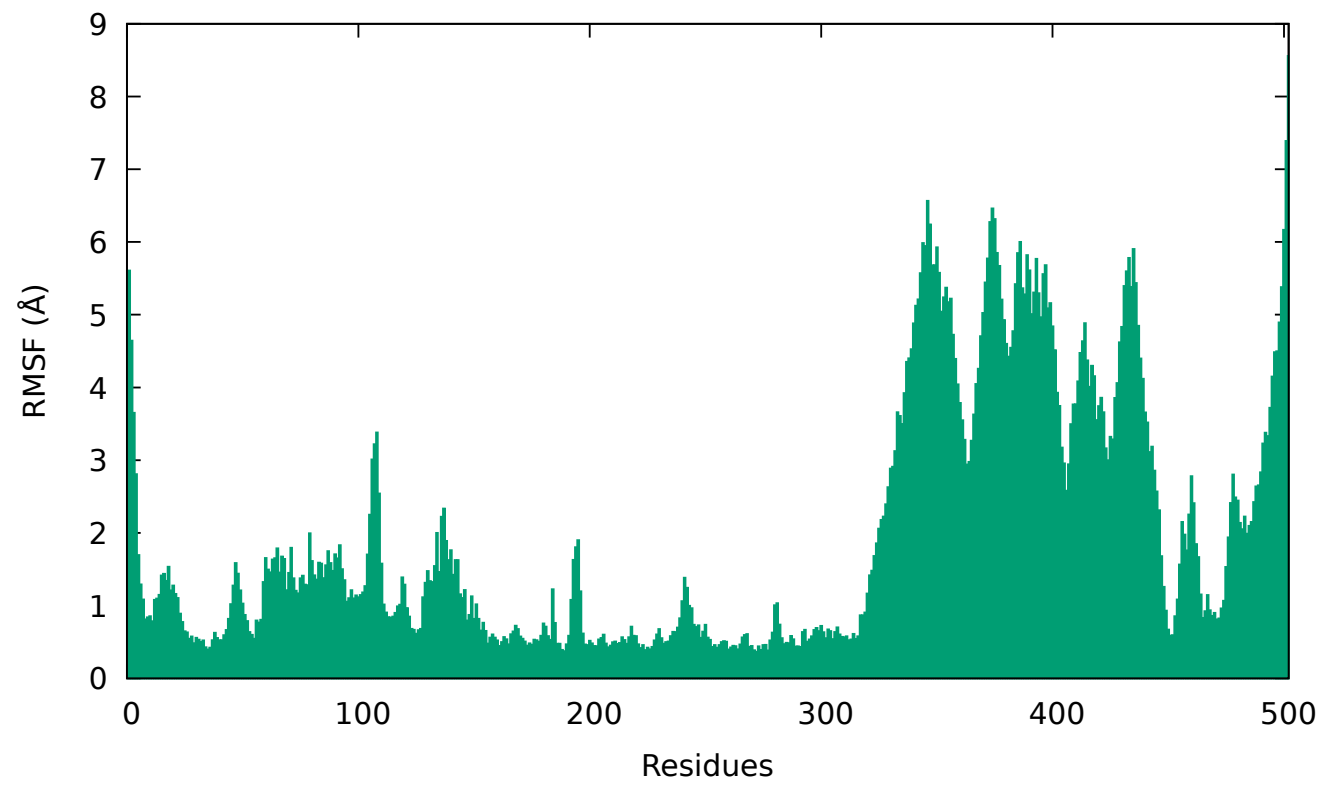

Figure S6: Mean RMSF of $\mathrm{C}_{\alpha}$-backbone atoms of each residue of DrBPhP monomer computed during the last $400 \mathrm{~ns}$ cMD simulation using AMBER ff14SB after alignment $\mathrm{w} / \mathrm{r} / \mathrm{t}$ the GAF domain.

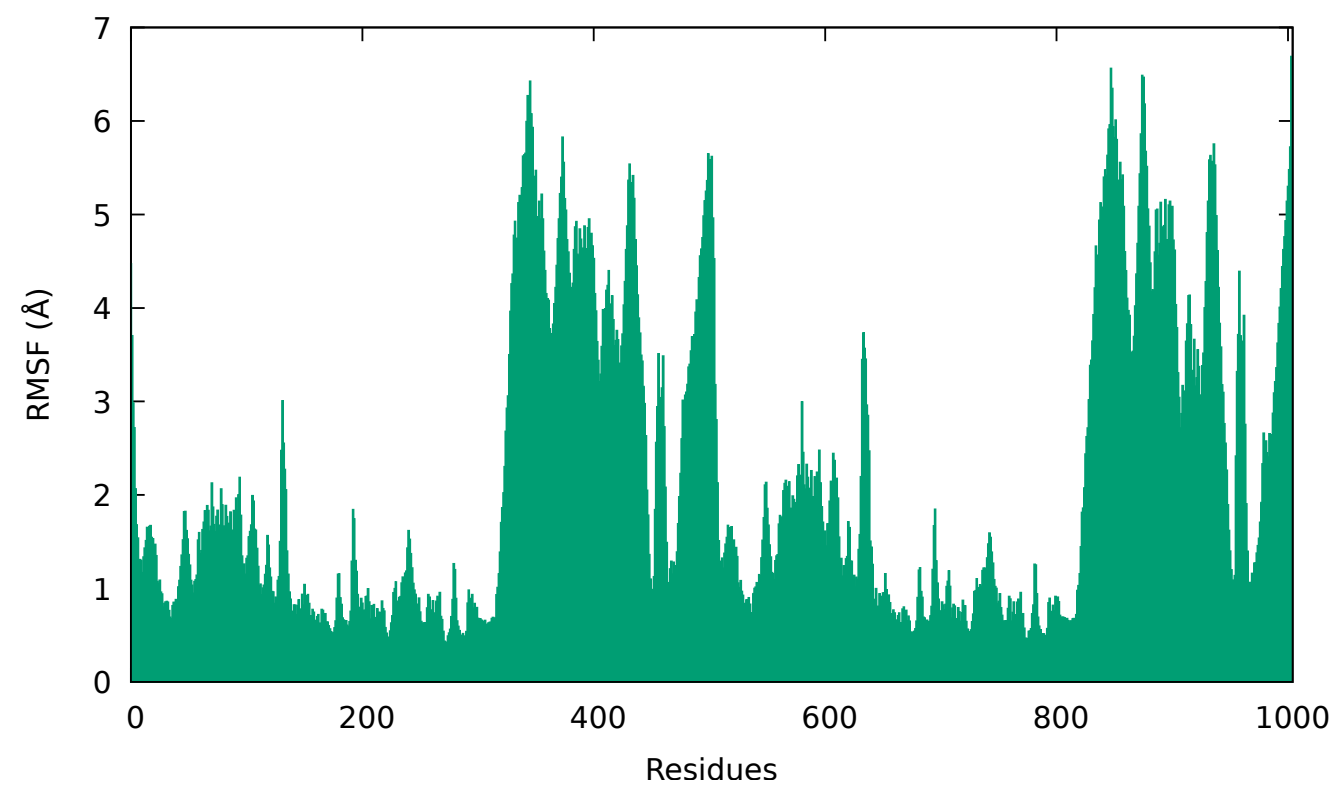

Figure S7: Mean RMSF of $\mathrm{C}_{\alpha}$-backbone atoms of each residue of $\mathrm{DrBPhP}$ dimer computed during the last $400 \mathrm{~ns}$ cMD simulation using CHARMM27 after alignment $\mathrm{w} / \mathrm{r} / \mathrm{t}$ the GAF domain. 


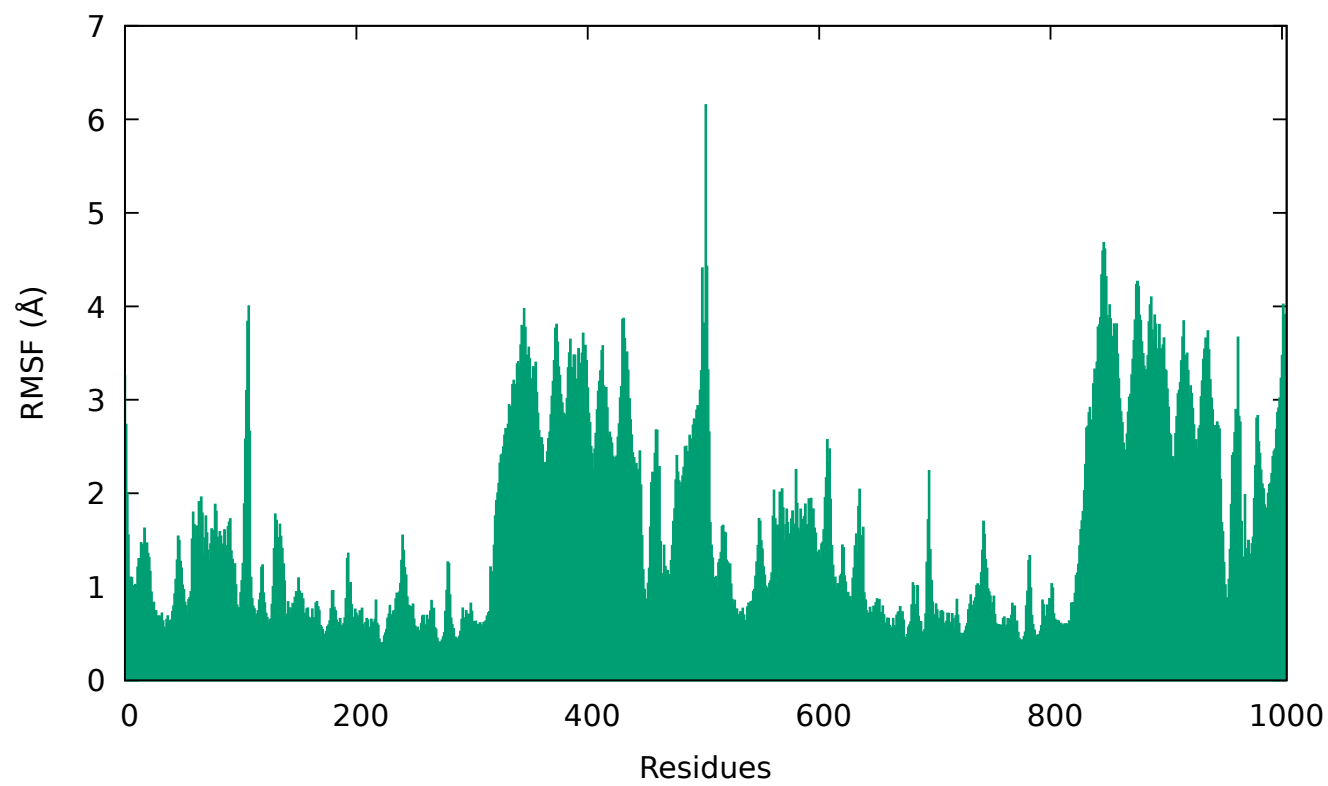

Figure S8: Mean RMSF of $\mathrm{C}_{\alpha}$-backbone atoms of each residue of $\mathrm{DrBPhP}$ dimer computed during the last $400 \mathrm{~ns}$ cMD simulation using AMBER ff14SB after alignment $\mathrm{w} / \mathrm{r} / \mathrm{t}$ the GAF domain.

As clearly visible in the RMSF graphs, the PHY domain residues (from 317 to 502) are the main contributor, beside the highly flexible loop regions, like the Nterminus. This underline the observed high flexibility of the PHY domain, pivoting around L311 in the $\alpha$-helical spine. RMSF values obtained with CHARMM27 and AMBER ff14SB force fields are comparable and reproducible. 


\section{Secondary structure plots}

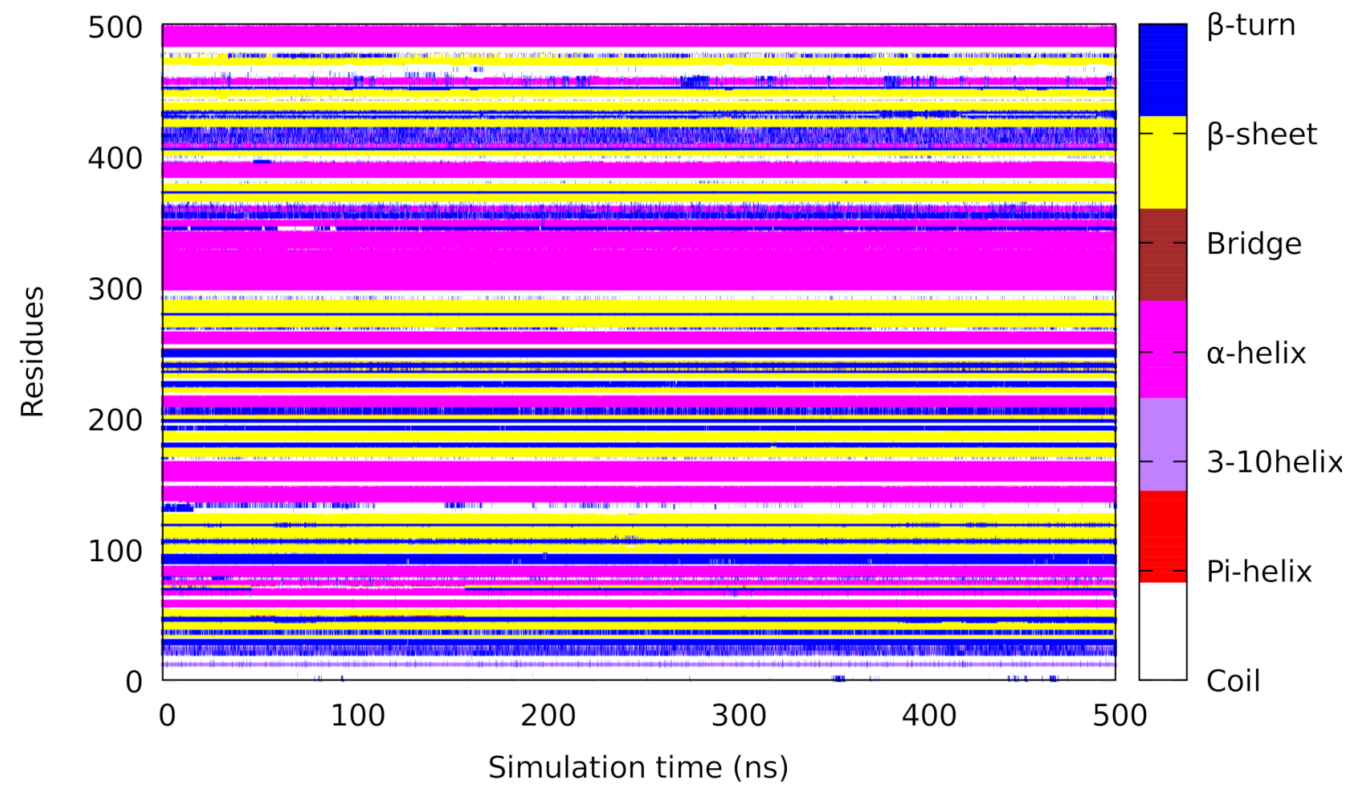

Figure S9: Secondary structure evolution plot of DrBphP monomer from cMD with CHARMM27.

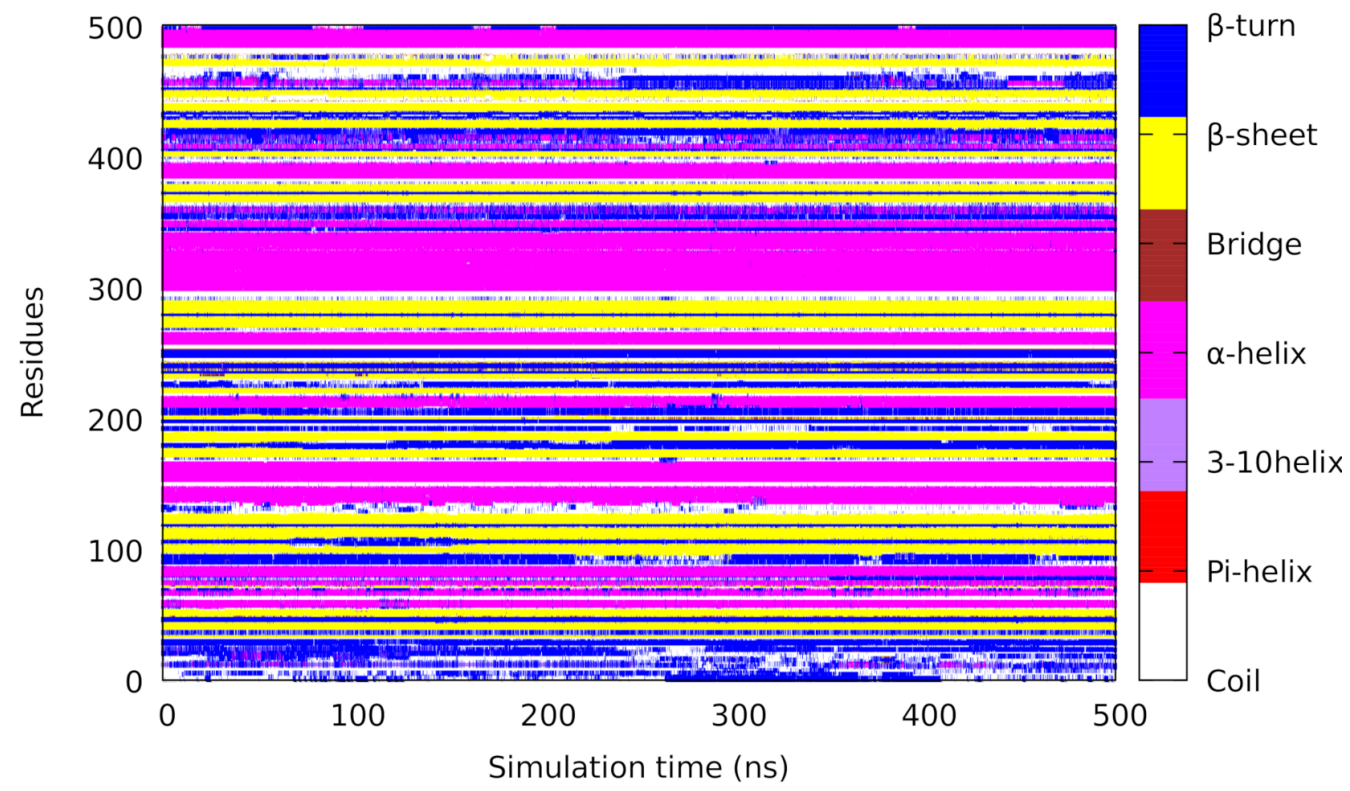

Figure S10: Secondary structure evolution plot of DrBphP monomer from aMD with CHARMM27. 


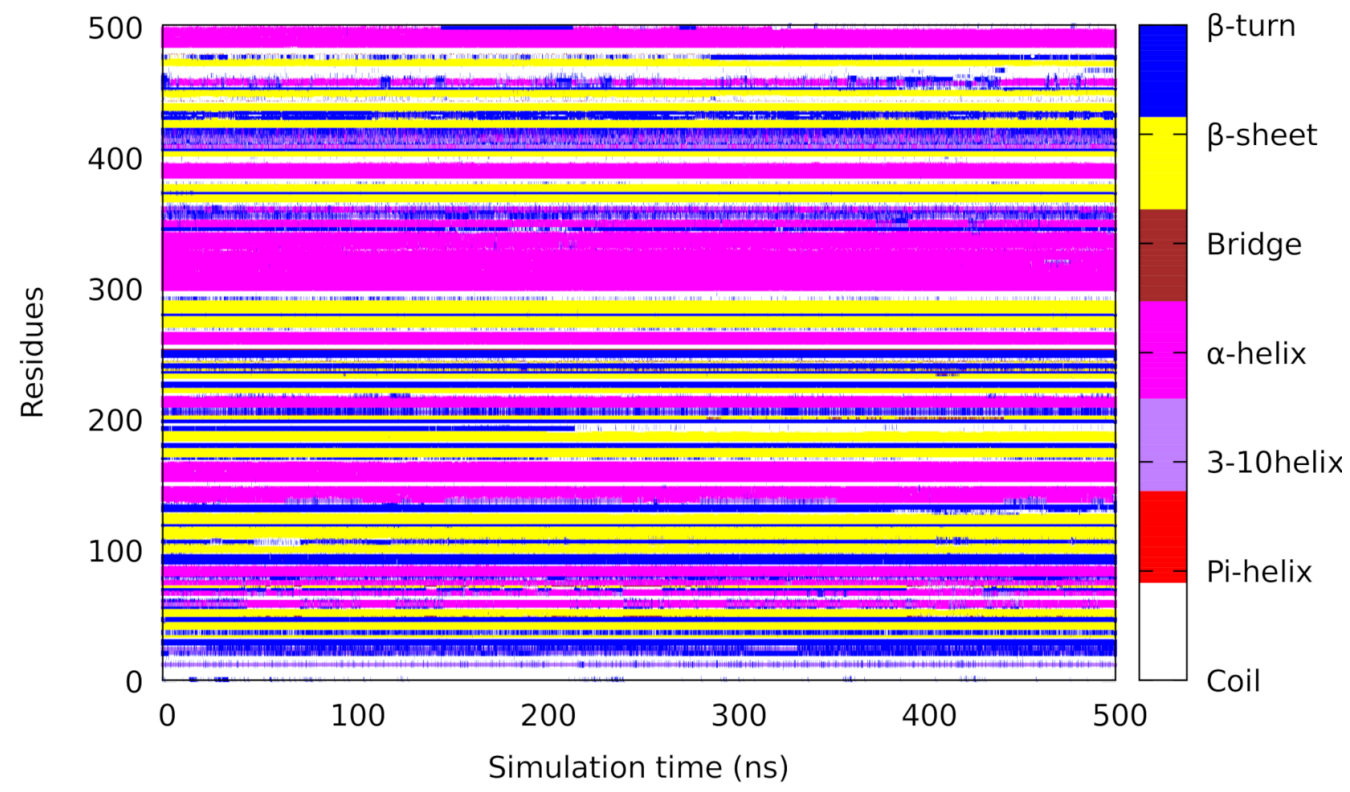

Figure S11: Secondary structure evolution plot of DrBphP monomer from cMD with AMBER ff14SB.

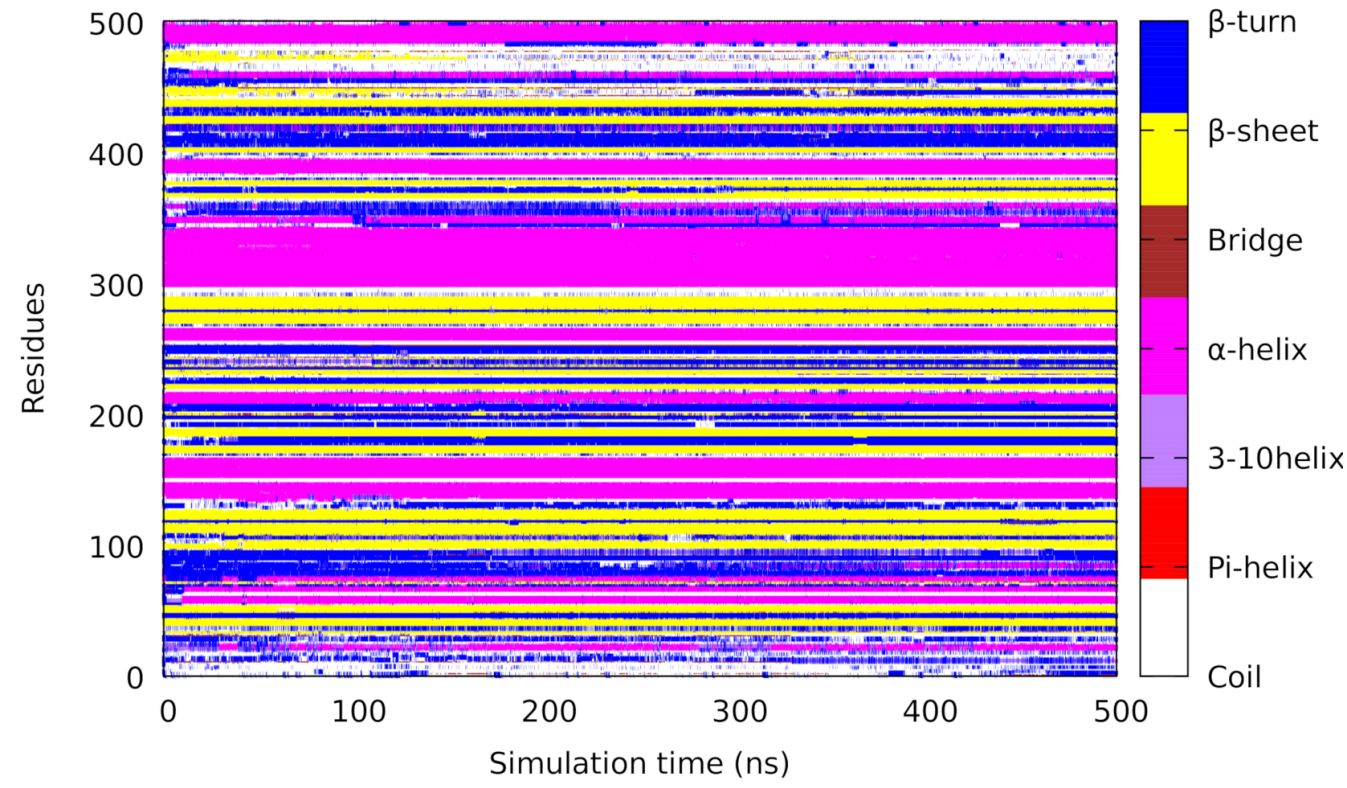

Figure S12: Secondary structure evolution plot of DrBphP monomer from GaMD with AMBER ff14SB. 


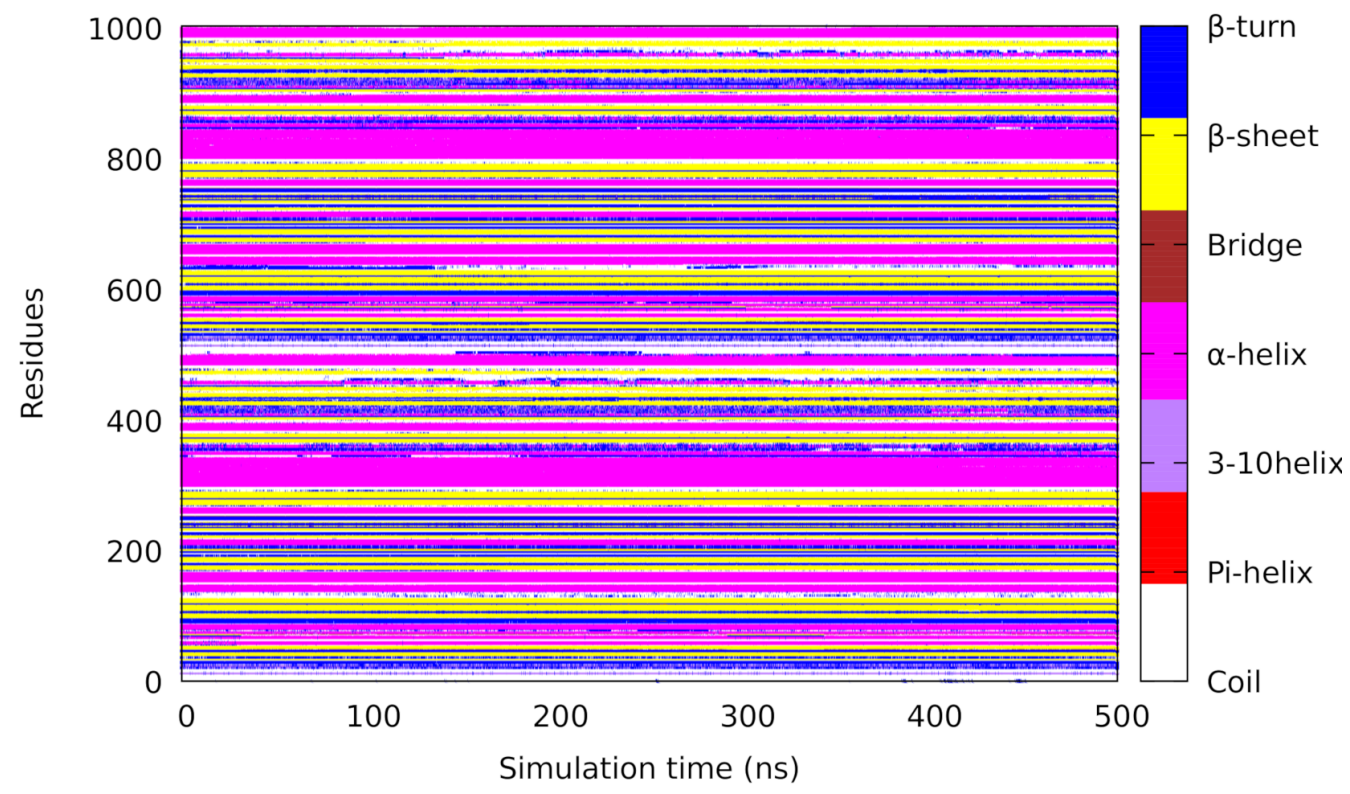

Figure S13: Secondary structure evolution plot of DrBphP dimer from cMD with CHARMM27

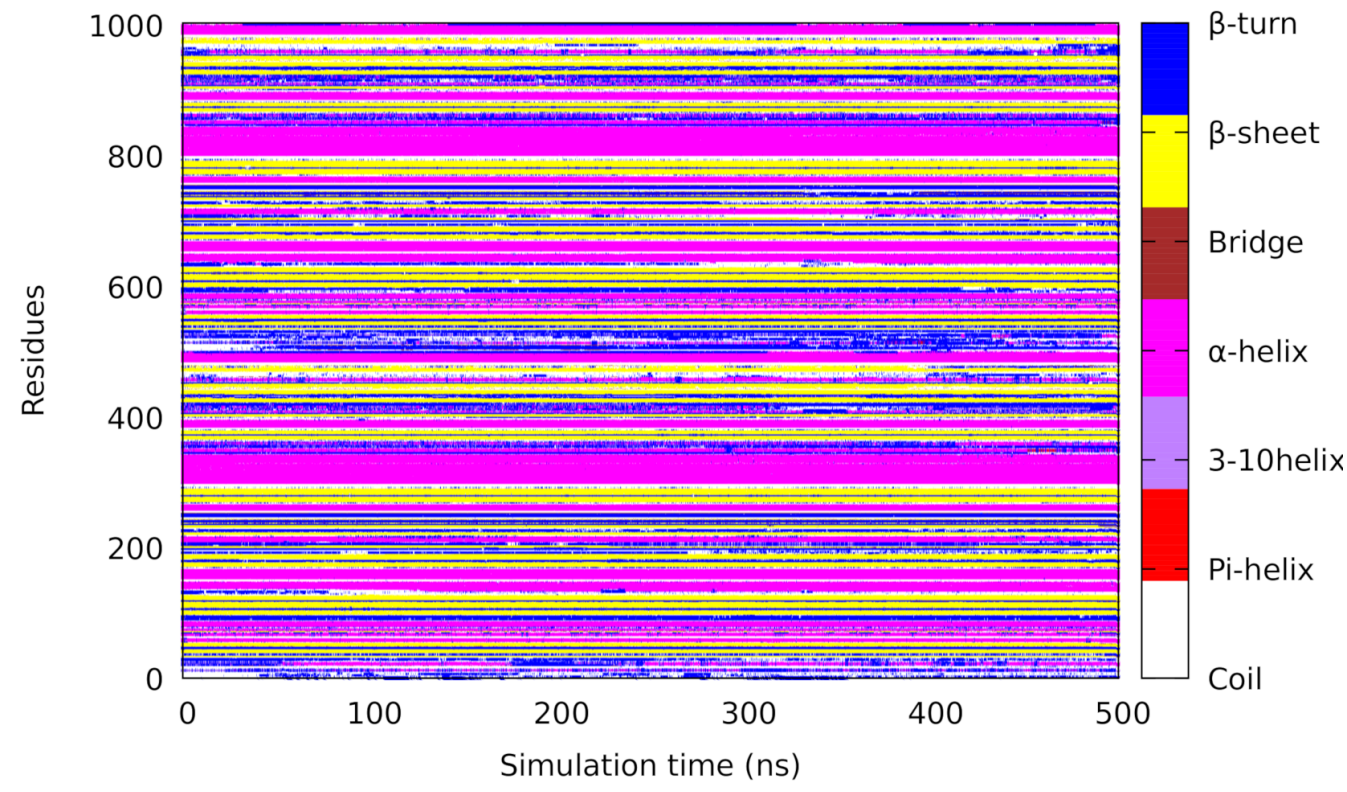

Figure S14: Secondary structure evolution plot of DrBphP dimer from aMD with CHARMM27. 


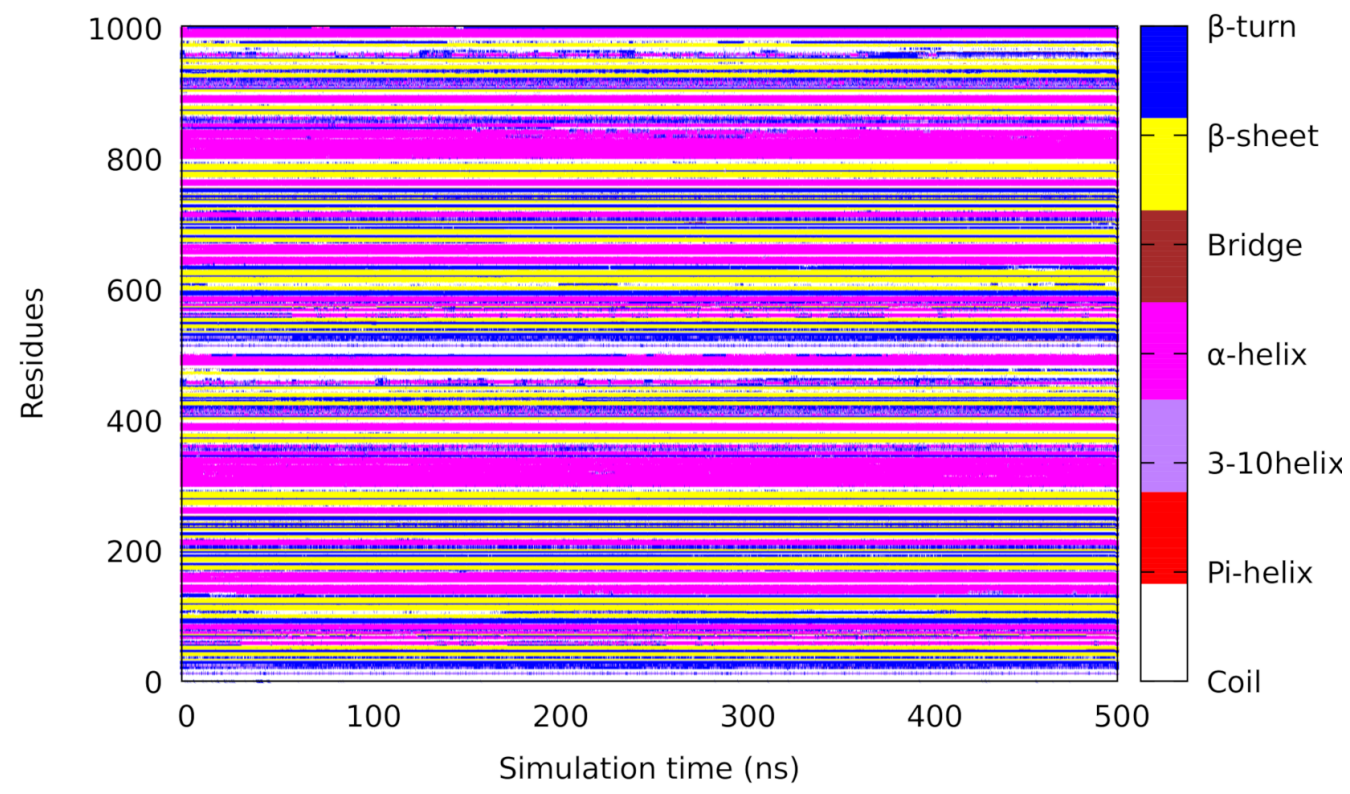

Figure S15: Secondary structure evolution plot of DrBphP dimer from cMD with AMBER ff14SB.

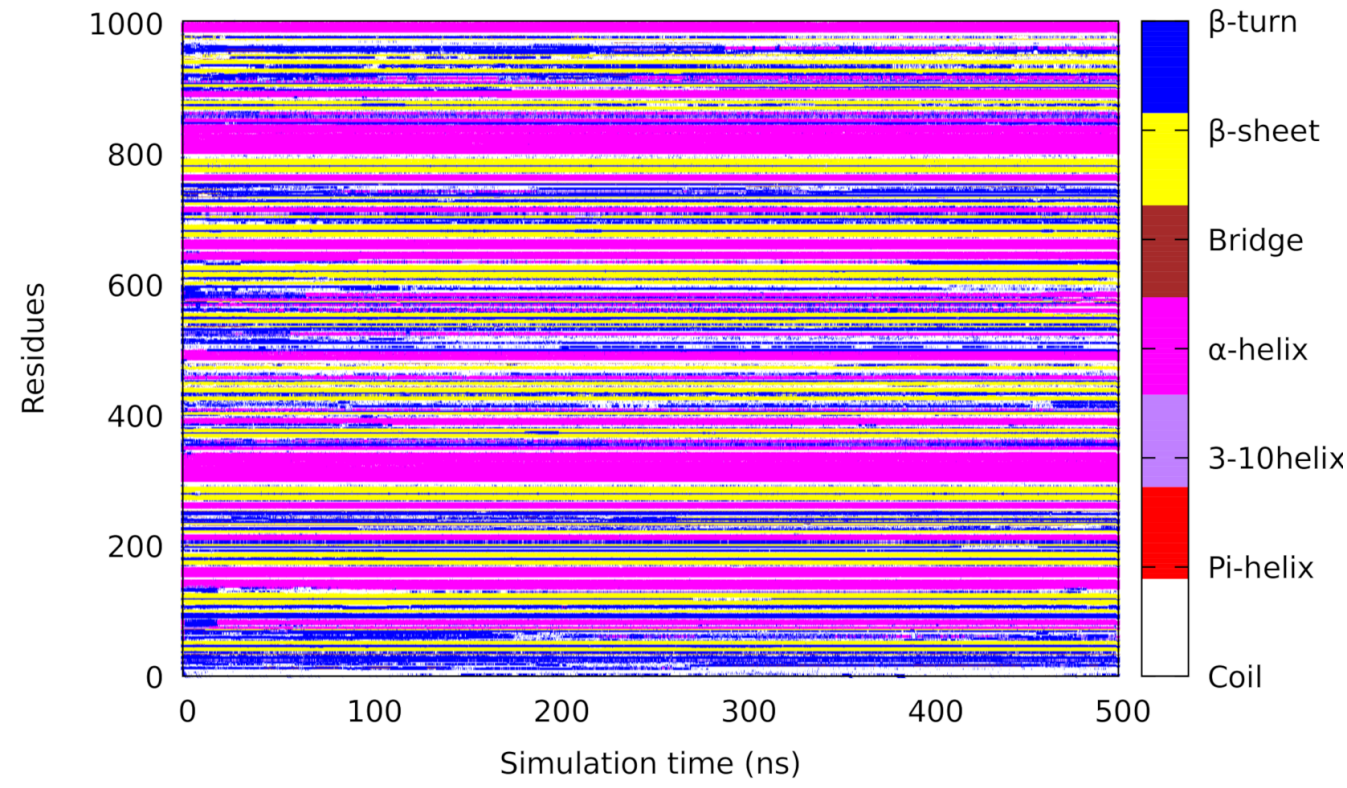

Figure S16: Secondary structure evolution plot of DrBphP dimer from GaMD with AMBER ff14SB.

In all secondary structure evolution plots no relevant secondary transition can be seen, with some fluctuations involving more flexible regions like loops and turns. These fluctuations are more pronounced, as expected, in accelerated MD plots. 


\section{Center of mass (COM)}

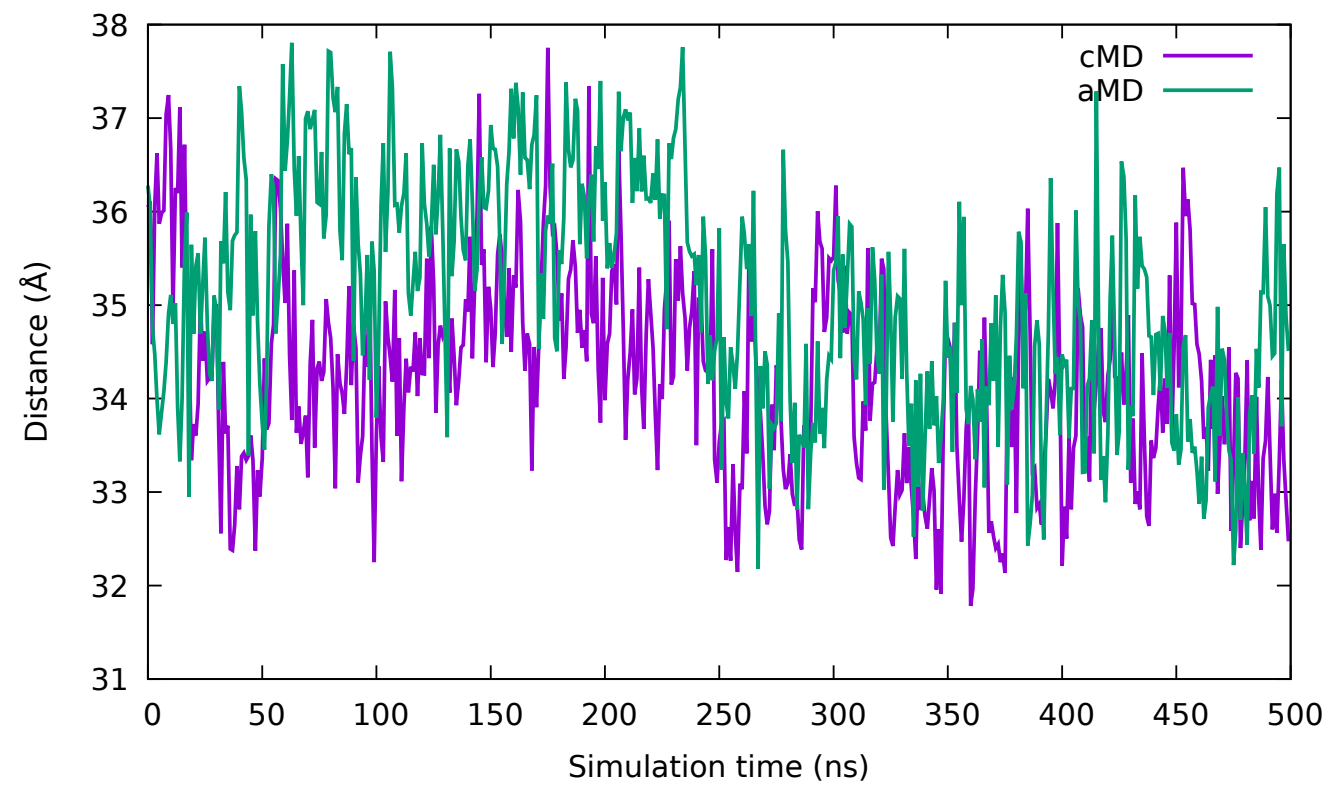

Figure S17: COM distance plot between GAF and PHY domains of the DrBphP monomer according to cMD (purple) and aMD (green) simulations with CHARMM27.

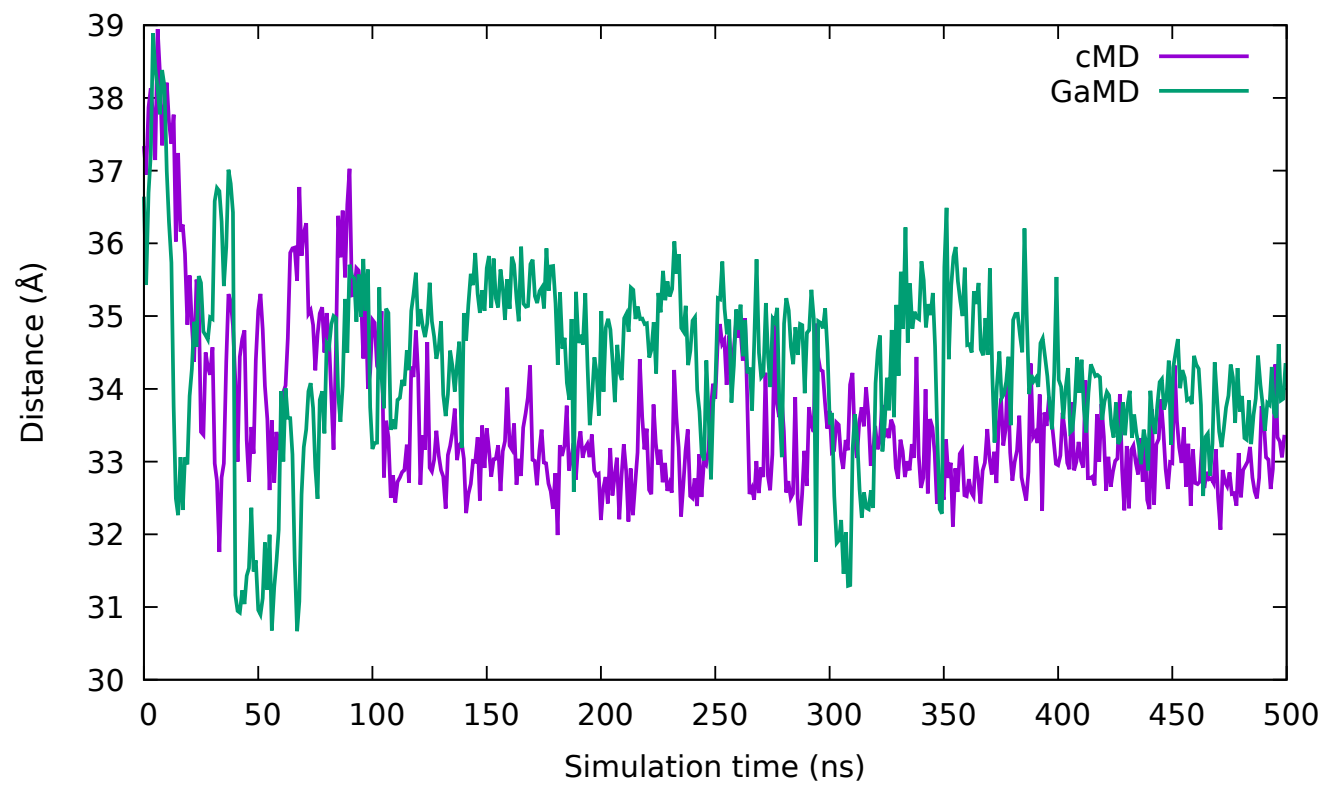

Figure S18: COM distance plot between GAF and PHY domains of the DrBphP monomer according to cMD (purple) and GaMD (green) simulations with AMBER ff14SB. 


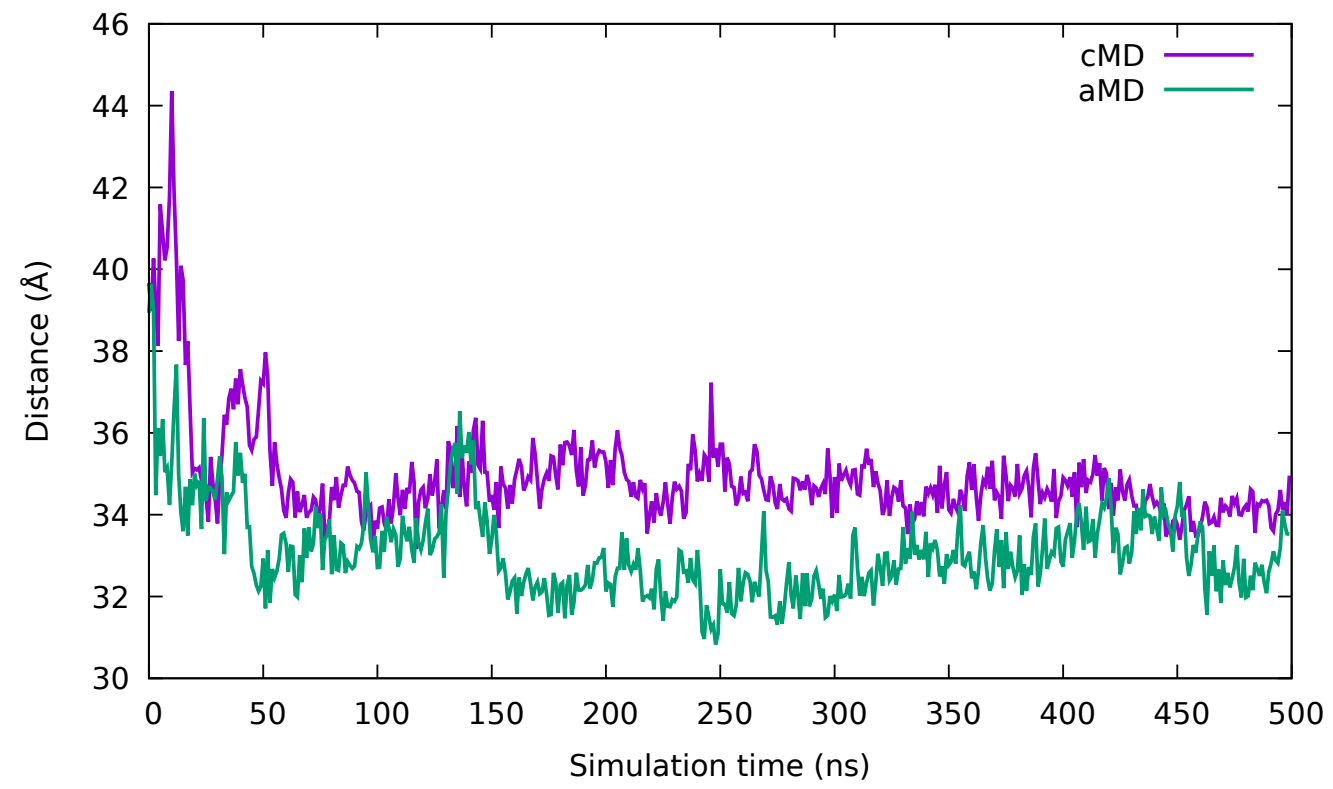

Figure S19: COM distance plot between two sister PHY domains of the DrBphP dimer according to cMD (purple) and aMD (green) simulations with CHARMM27.

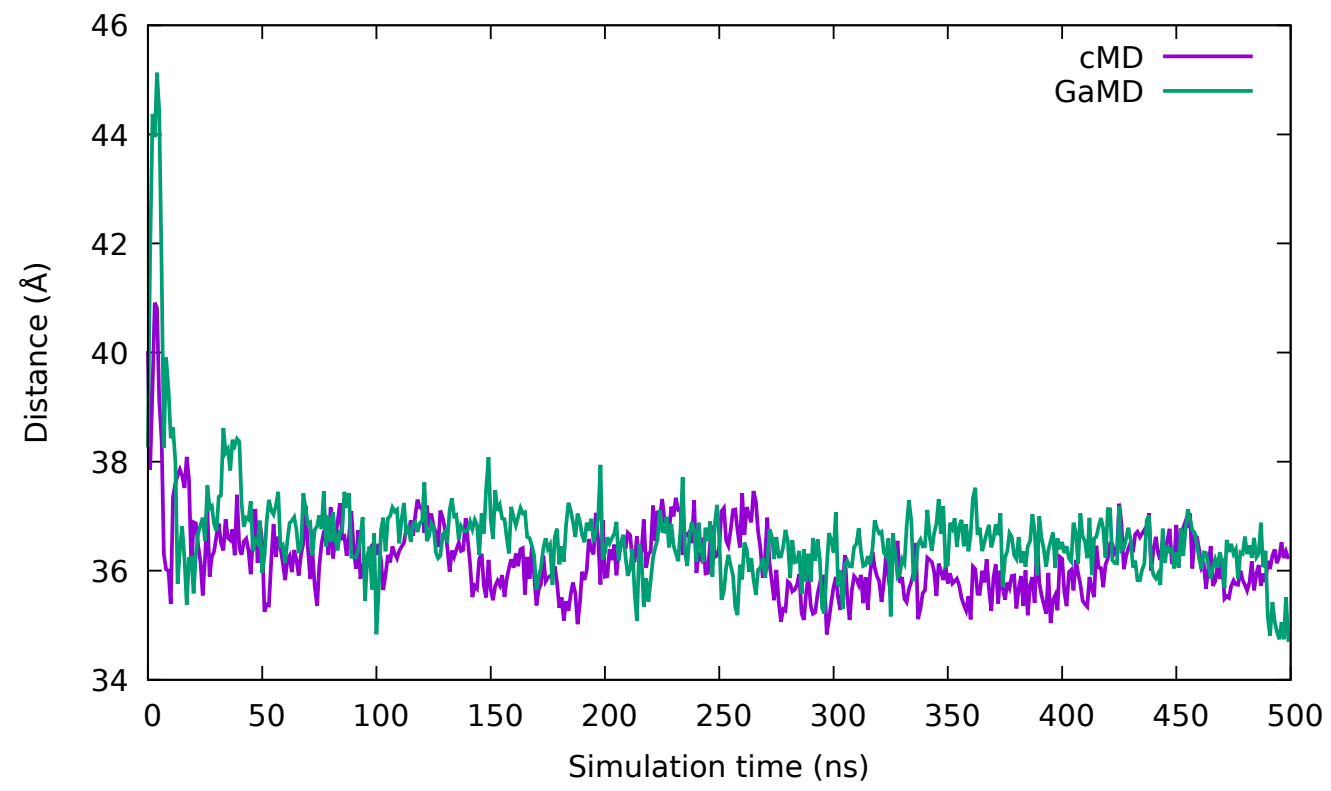

Figure S20: COM distance plot between two sister PHY domains of the DrBphP dimer according to cMD (purple) and GaMD (green) simulations with AMBER ff14SB. 


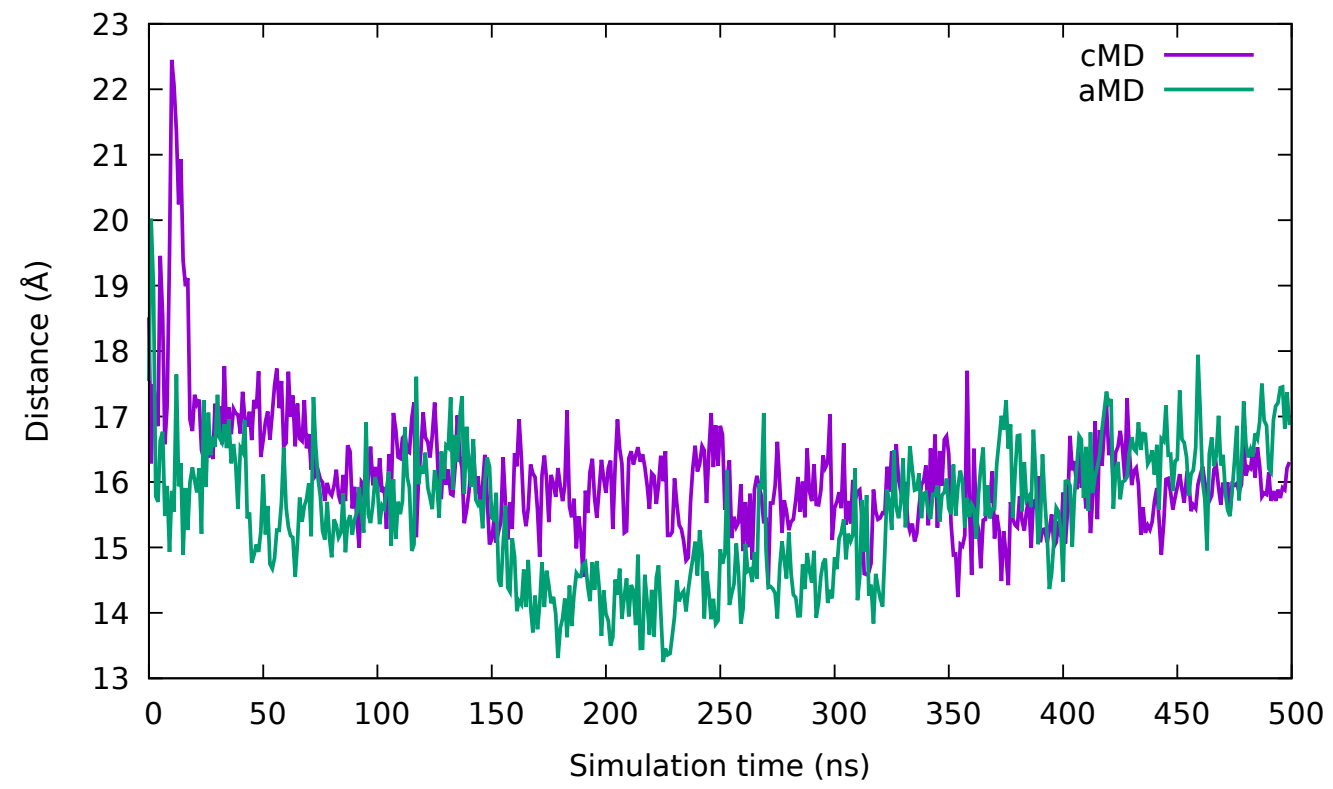

Figure S21: COM distance plot between two sister PHY domains of the DrBphP dimer according to cMD (purple) and aMD (green) simulations with CHARMM27 taking as reference the distance between the centres of mass of the two C-terminal helices (residues 484-502).

The two PHY domains approach each other very soon and they stay at the same distance throughout the rest of the simulation. AMBER ff14SB simulations are not very noisy and there is good agreement between classical and accelerated MD, with a final distance around $36 \AA$. CHARMM27 simulations are noisier and the final distance is around 34 Ainstead. 


\section{Dynamic Cross Correlation (DCC)}

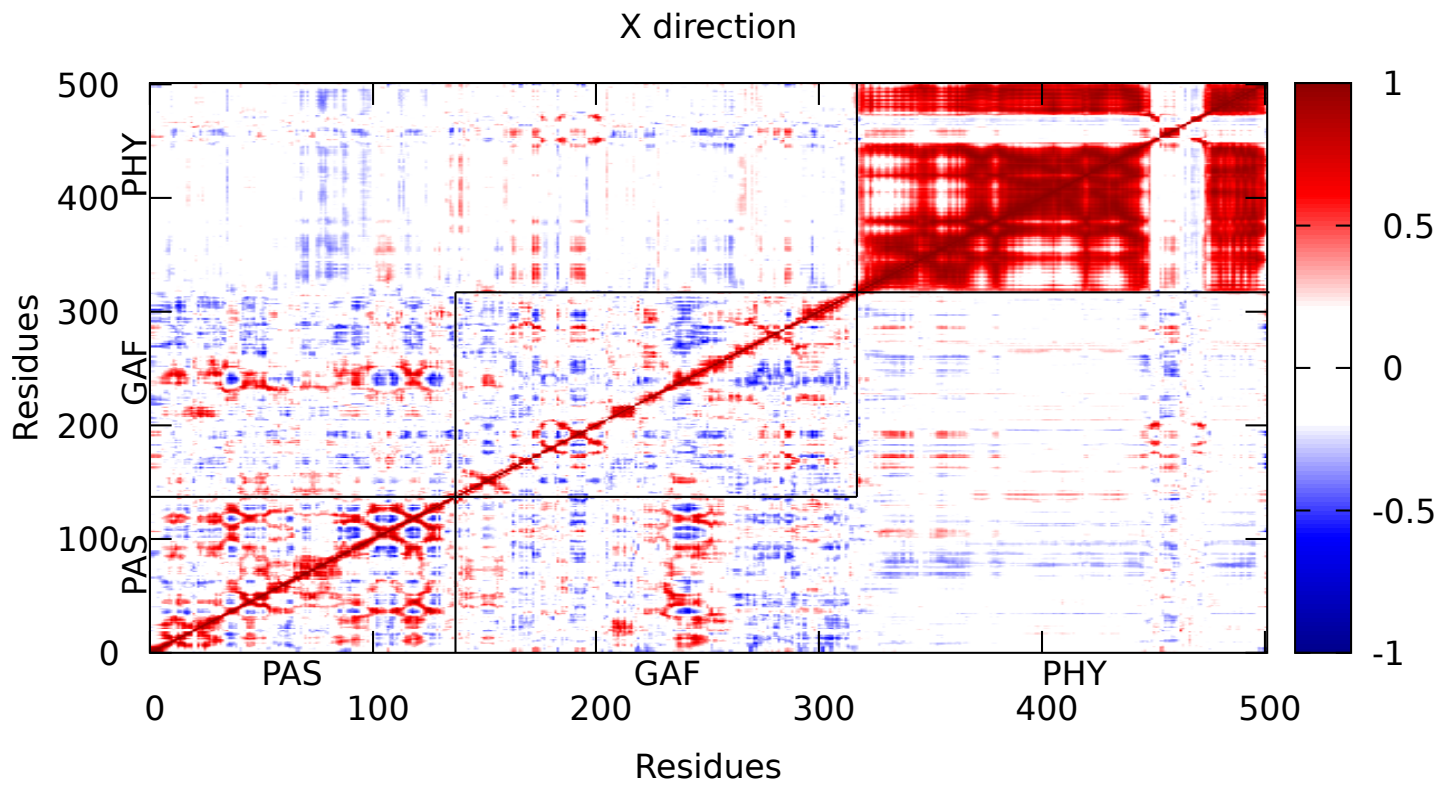

Figure S22: DCC along the $\mathrm{x}$ direction for the DrBphP monomer from cMD with AMBER ff14SB.

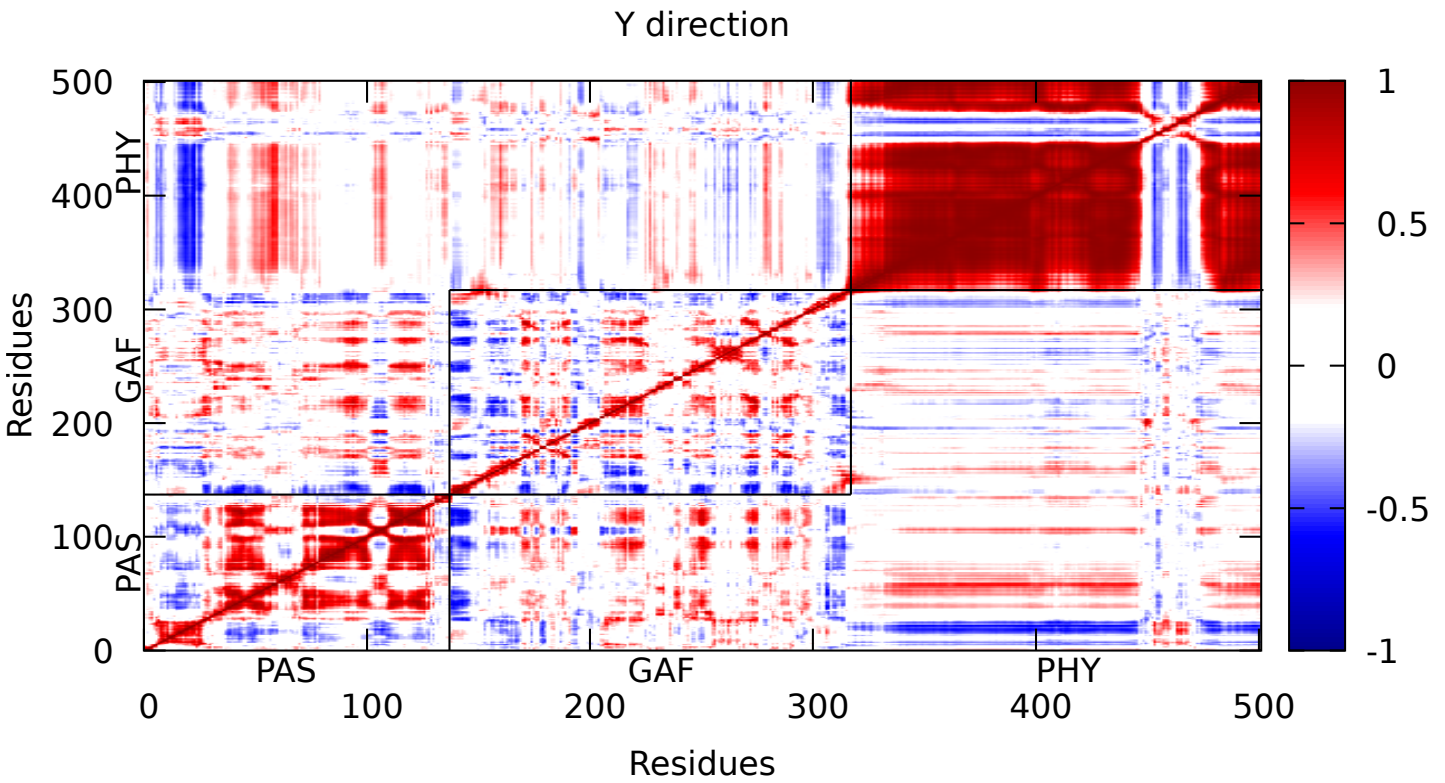

Figure S23: DCC along the y direction for the DrBphP monomer from cMD with AMBER ff14SB. 
Z direction

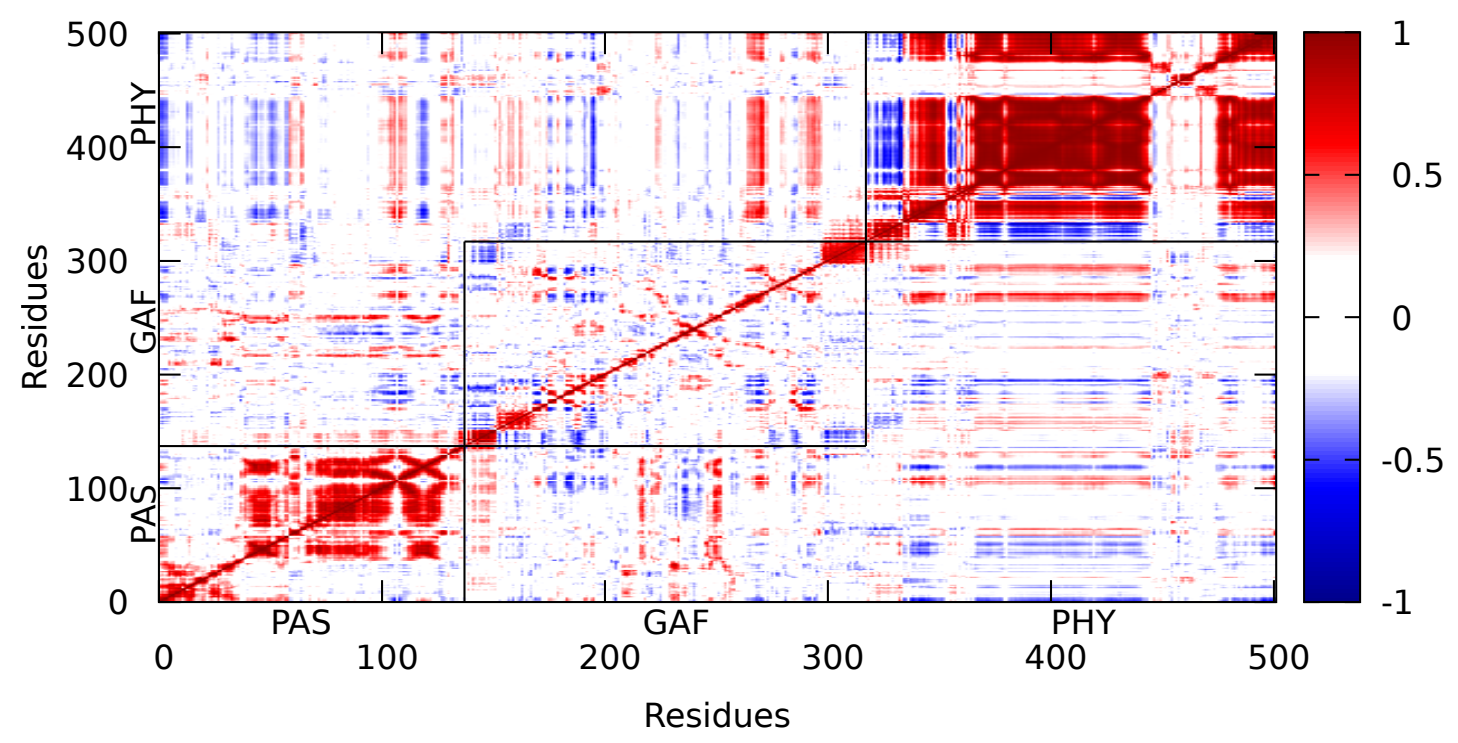

Figure S24: DCC along the $\mathrm{z}$ direction for the DrBphP monomer from cMD with AMBER ff14SB.

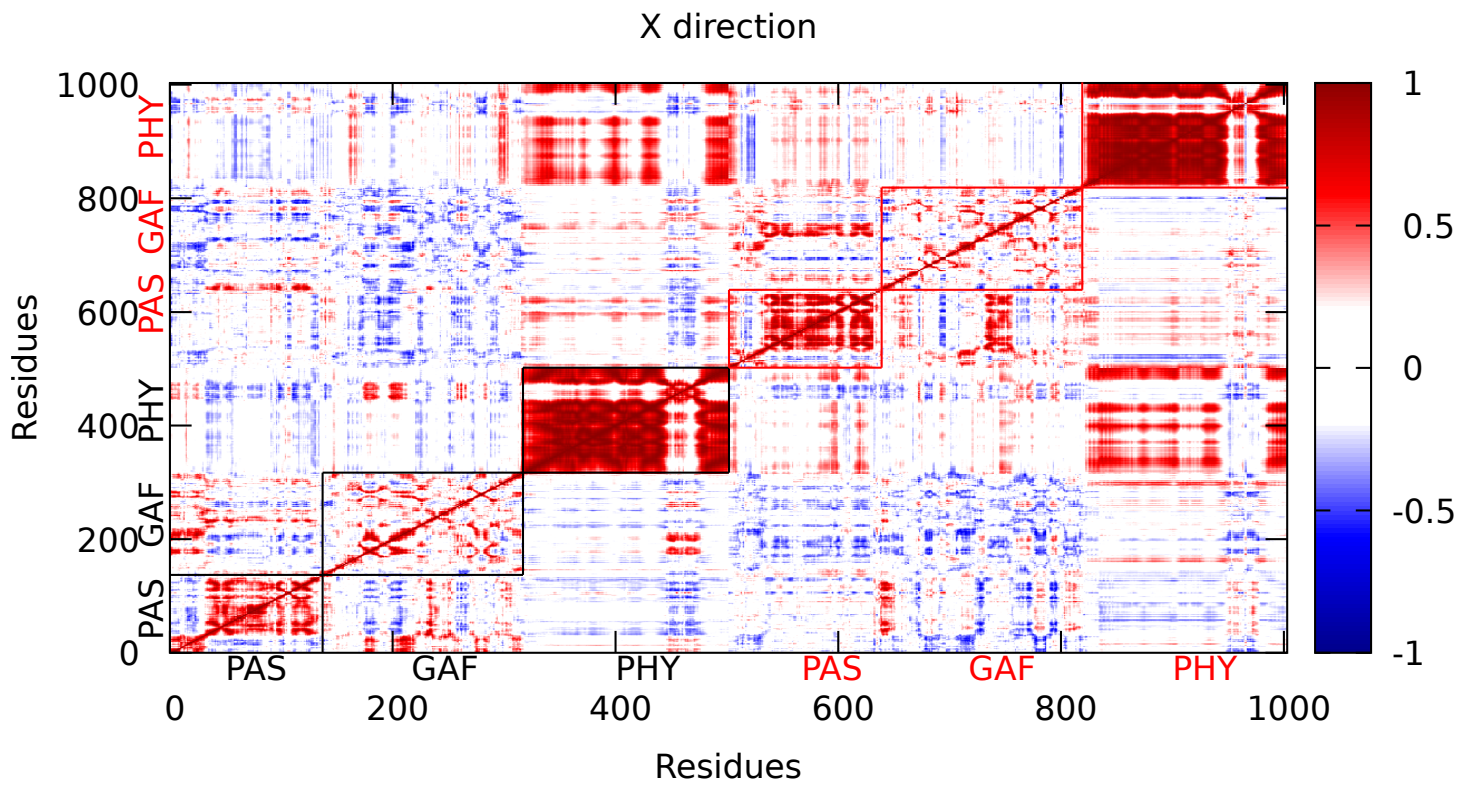

Figure S25: DCC along the x direction for the DrBphP dimer from cMD with AMBER ff14SB. 


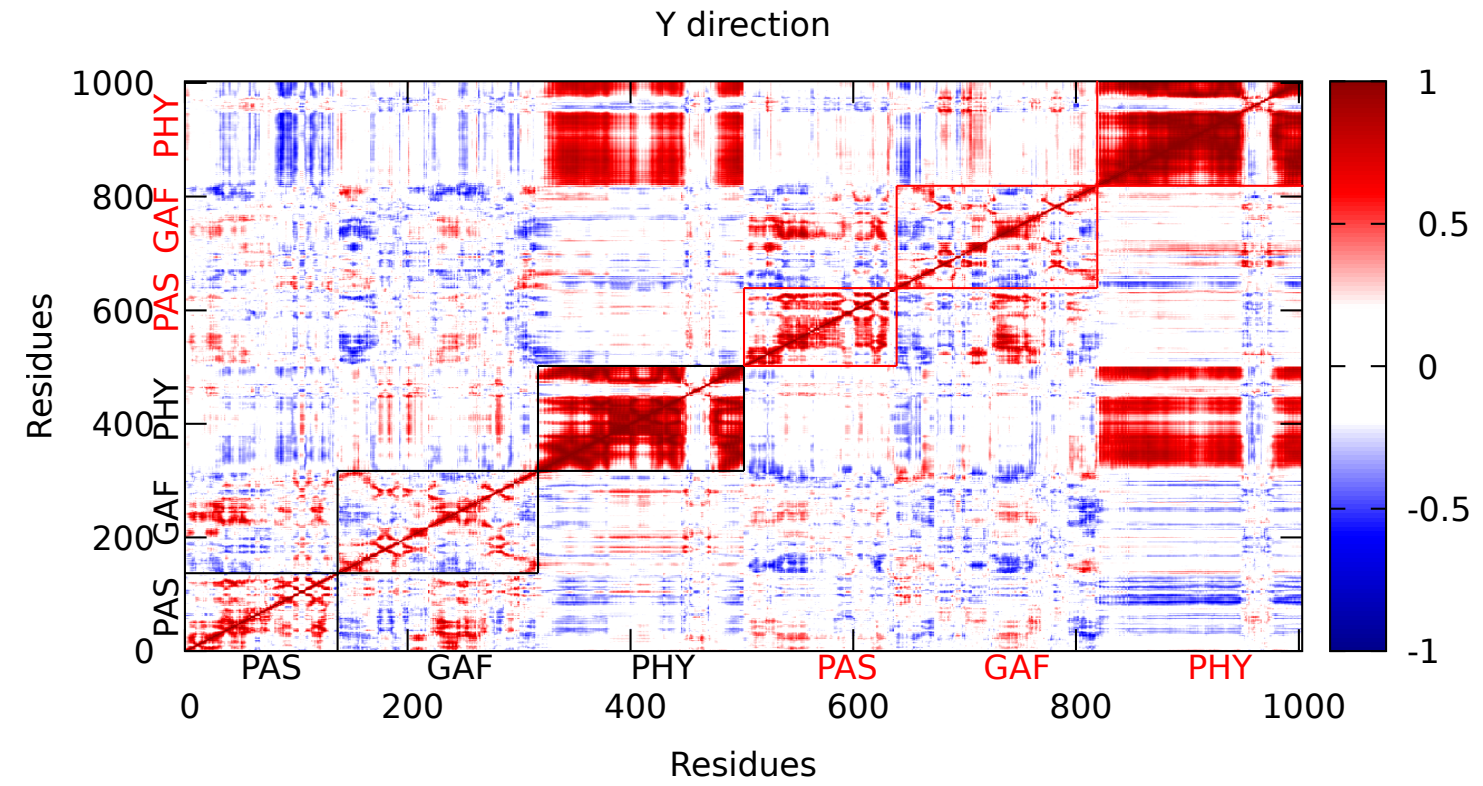

Figure S26: DCC along the y direction for the DrBphP dimer from cMD with AMBER ff14SB.

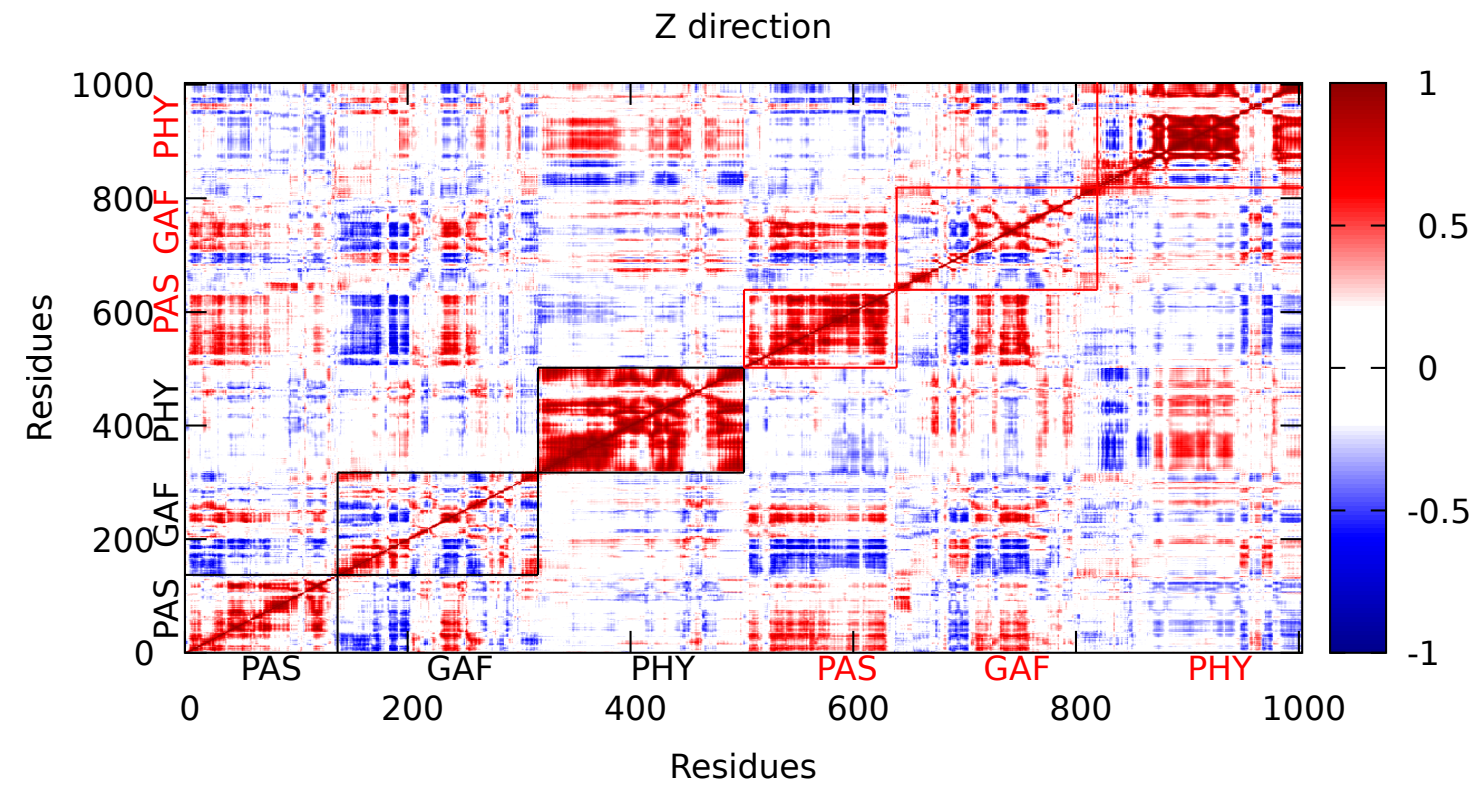

Figure S27: DCC along the $\mathrm{z}$ direction for the DrBphP dimer from cMD with AMBER ff14SB. 


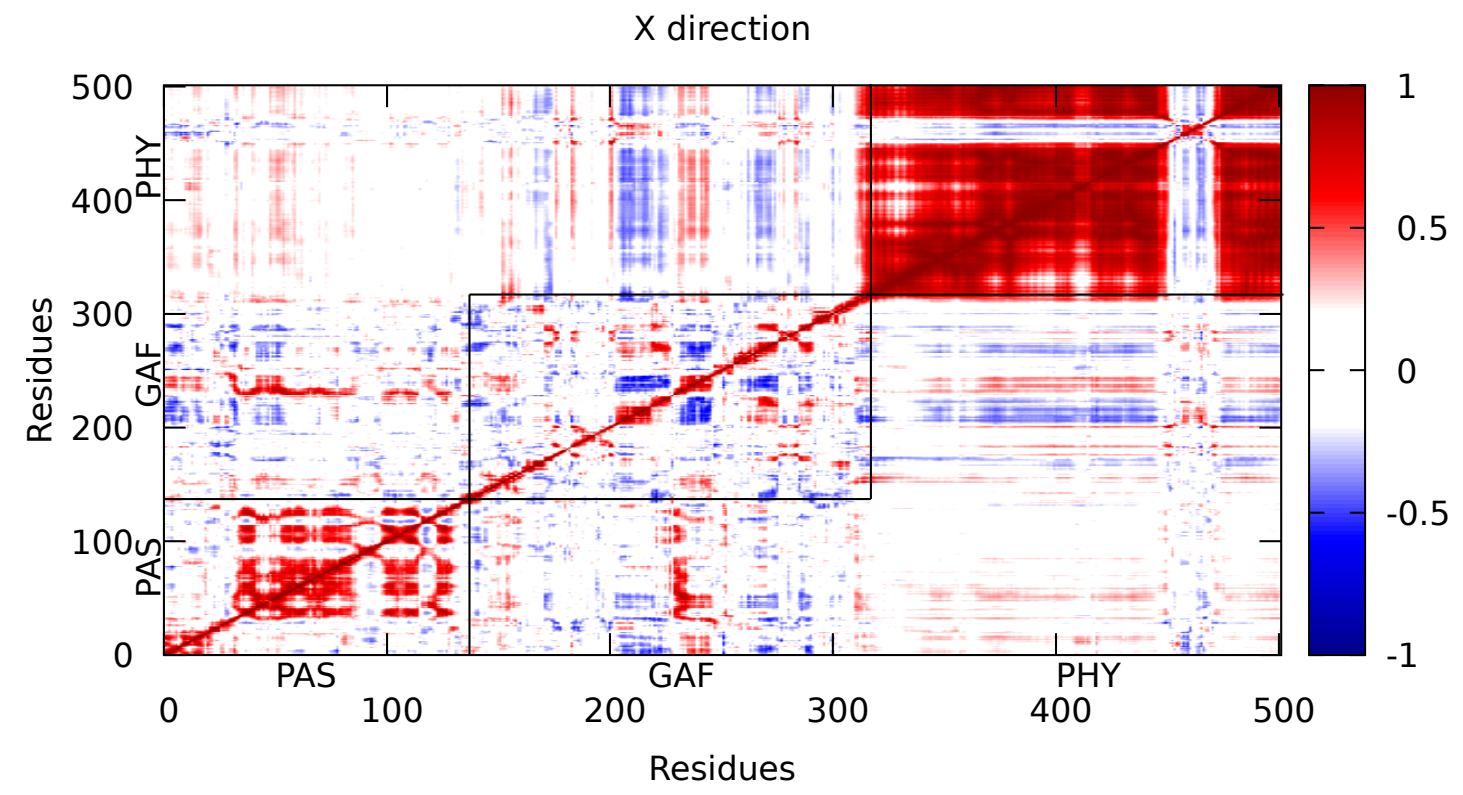

Figure S28: DCC along the $\mathrm{x}$ direction for the DrBphP monomer from aMD with CHARMM27.

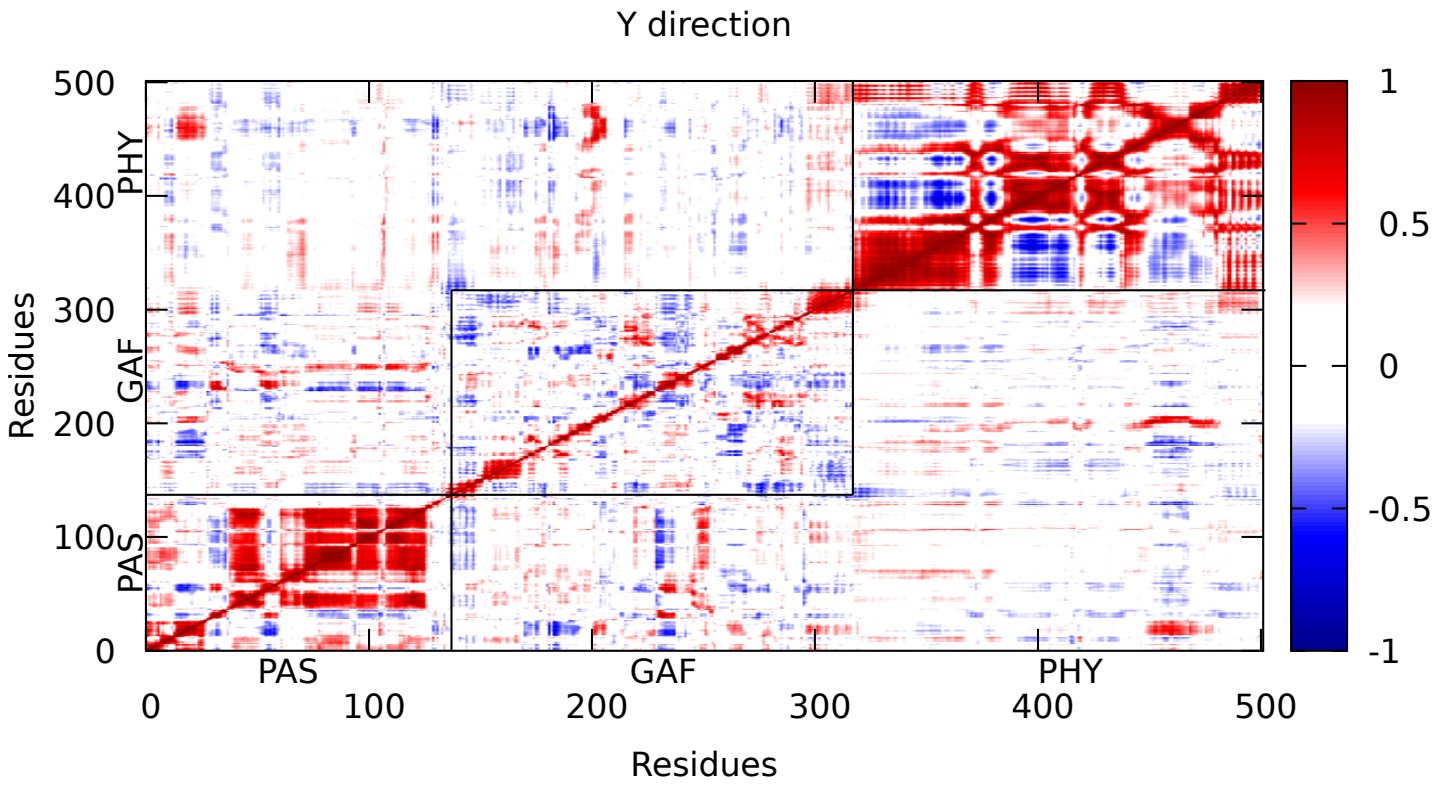

Figure S29: DCC along the y direction for the DrBphP monomer from aMD with CHARMM27. 
Z direction

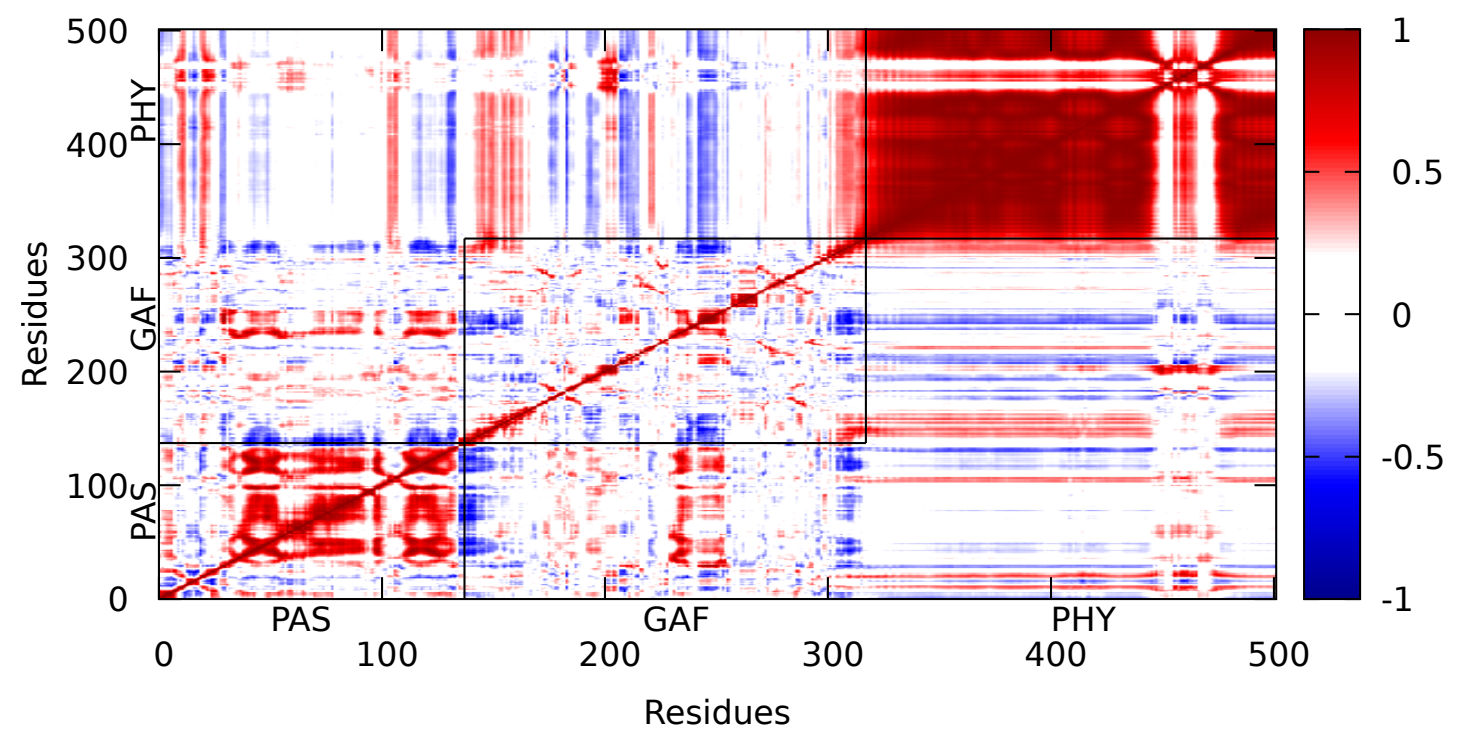

Figure S30: DCC along the $\mathrm{z}$ direction for the DrBphP monomer from aMD with CHARMM27.

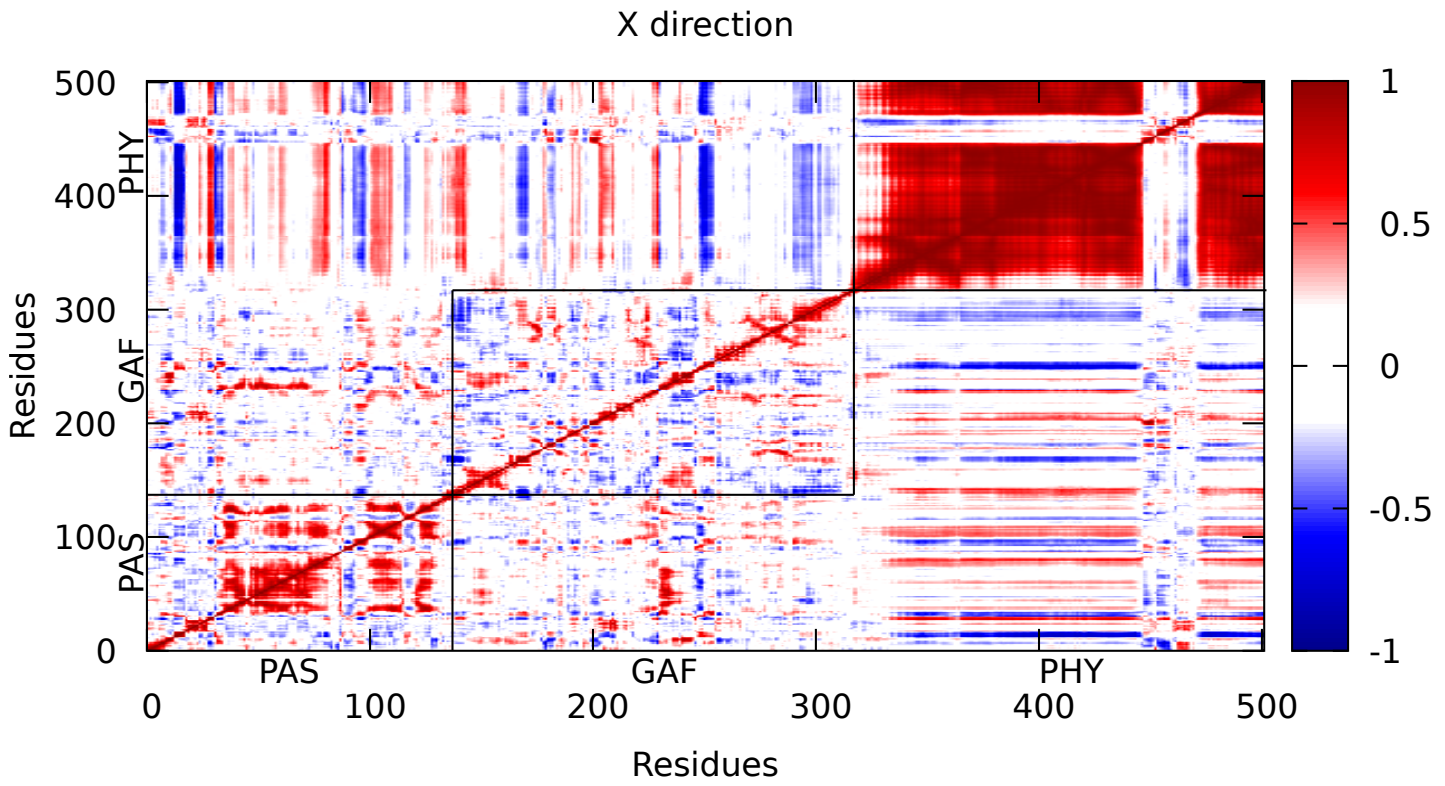

Figure S31: DCC along the x direction for the DrBphP monomer from GaMD with AMBER ff14SB. 
Y direction

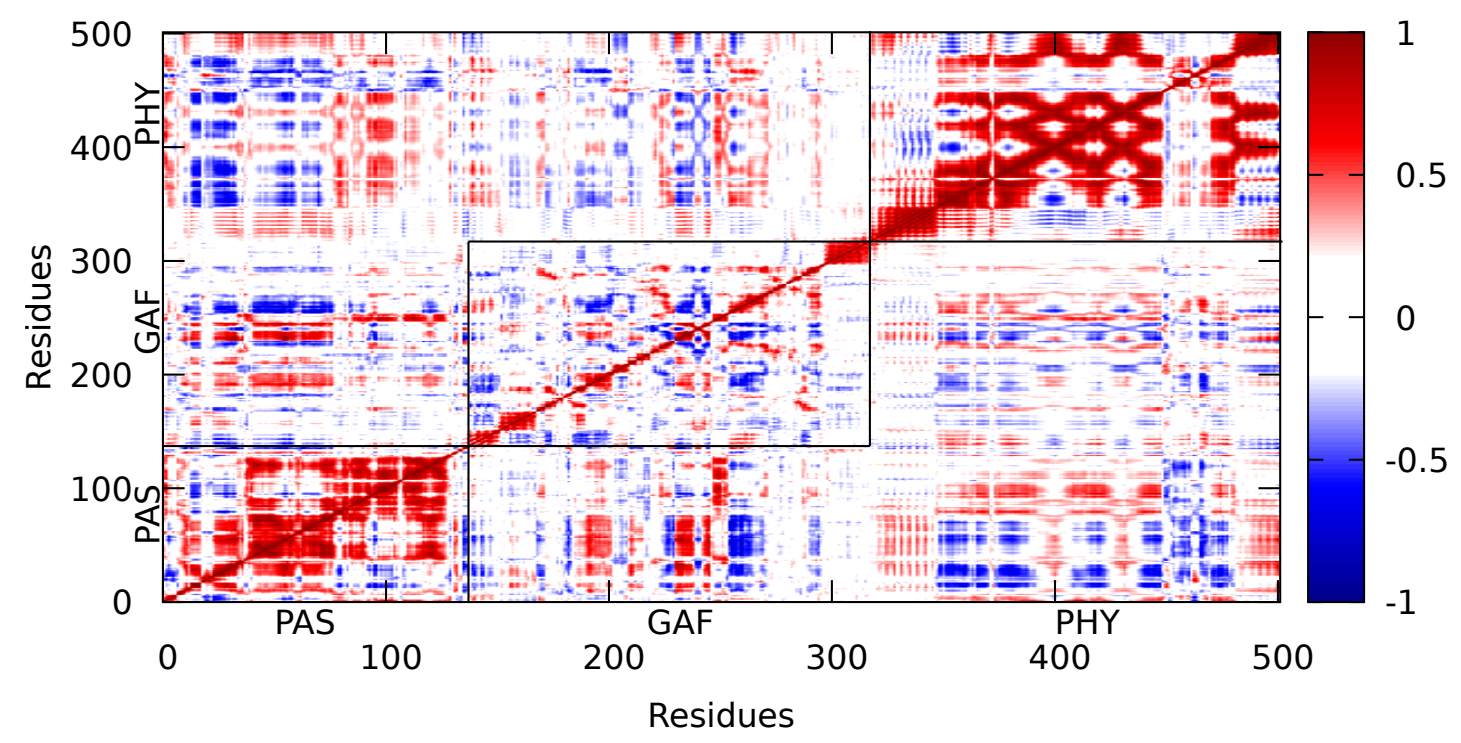

Figure S32: DCC along the y direction for the DrBphP monomer from GaMD with AMBER ff14SB.

\section{Z direction}

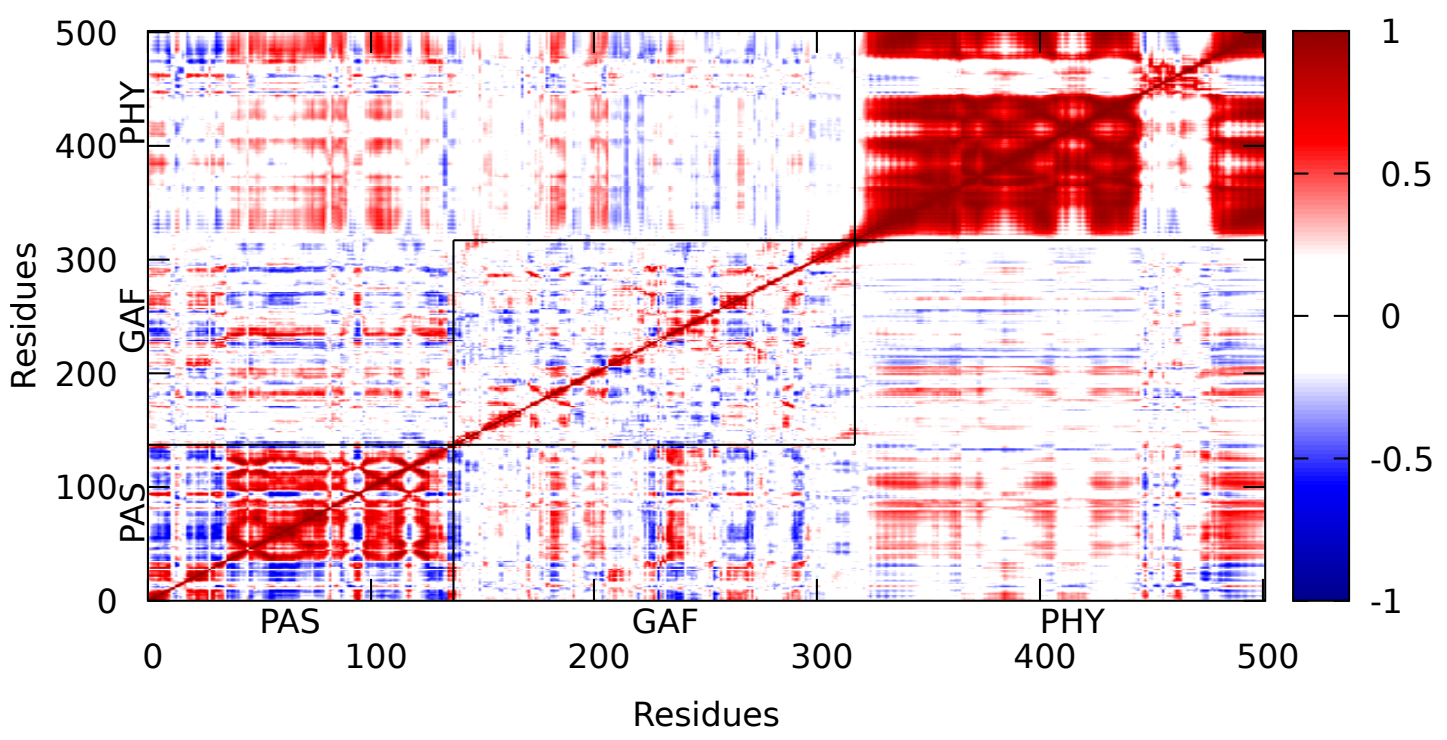

Figure S33: DCC along the $\mathrm{z}$ direction for the DrBphP monomer from GaMD with AMBER ff14SB. 


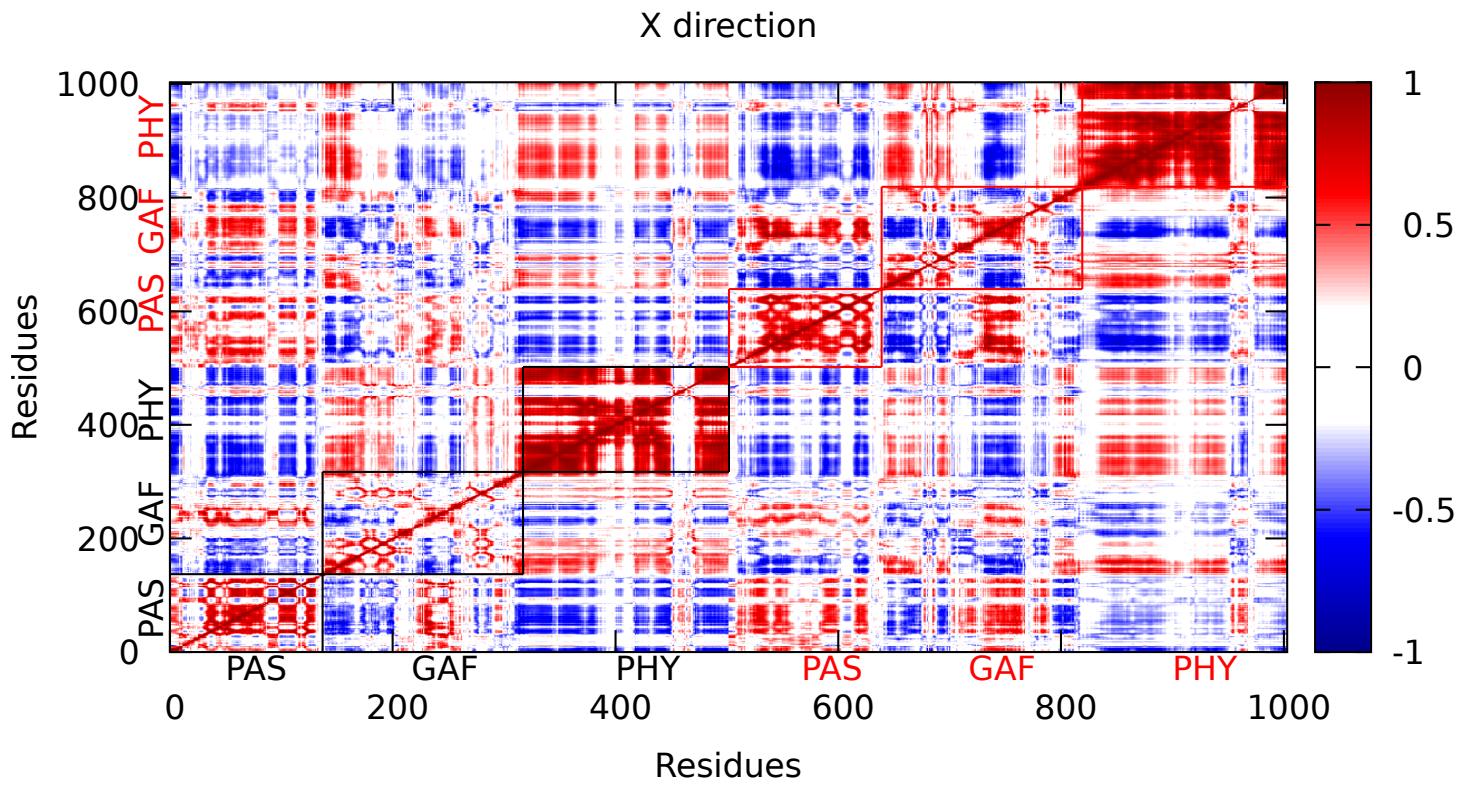

Figure S34: DCC along the $\mathrm{x}$ direction for the DrBphP dimer from aMD with CHARMM 27.

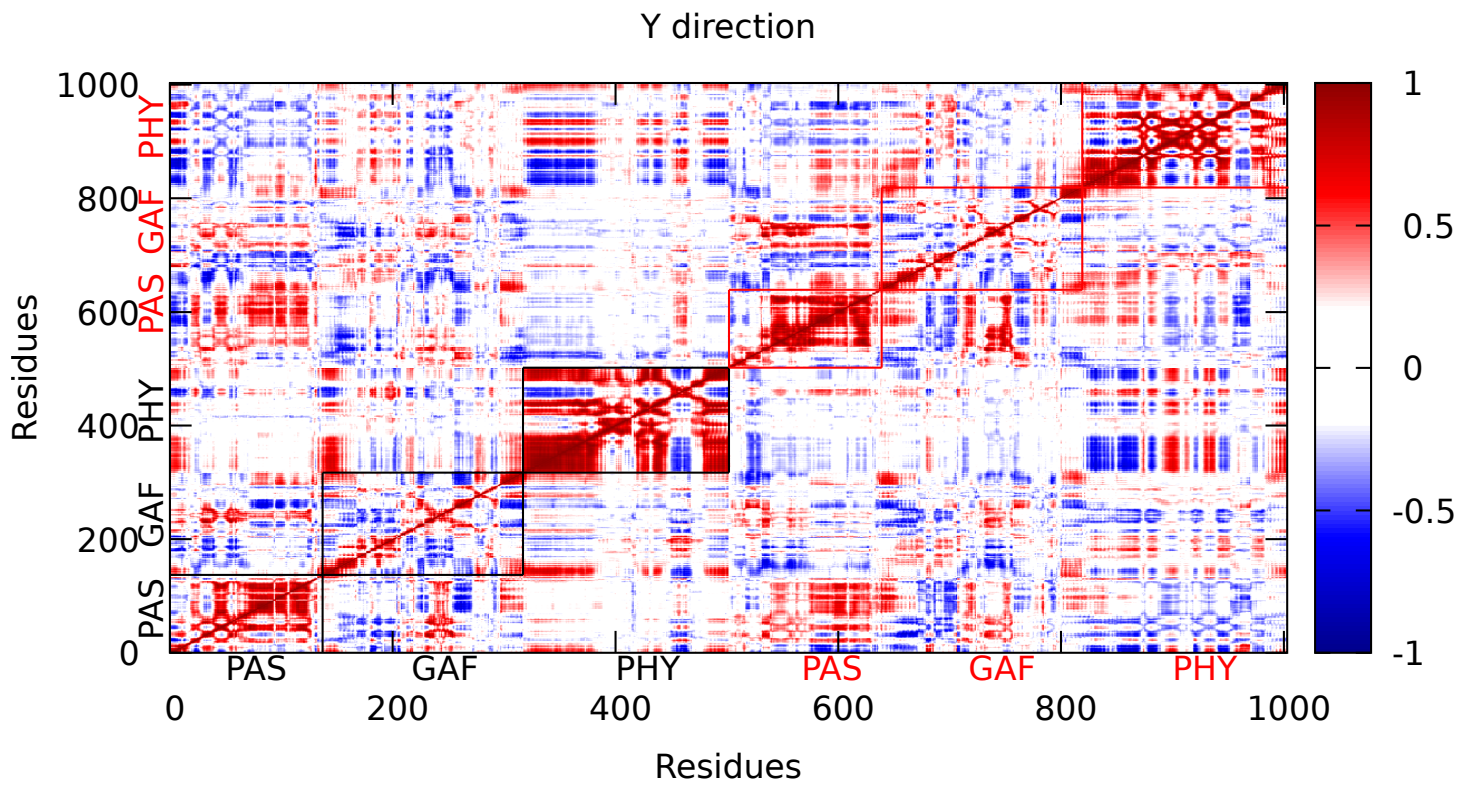

Figure S35: DCC along the y direction for the DrBphP dimer from aMD with CHARMM 27. 
Z direction

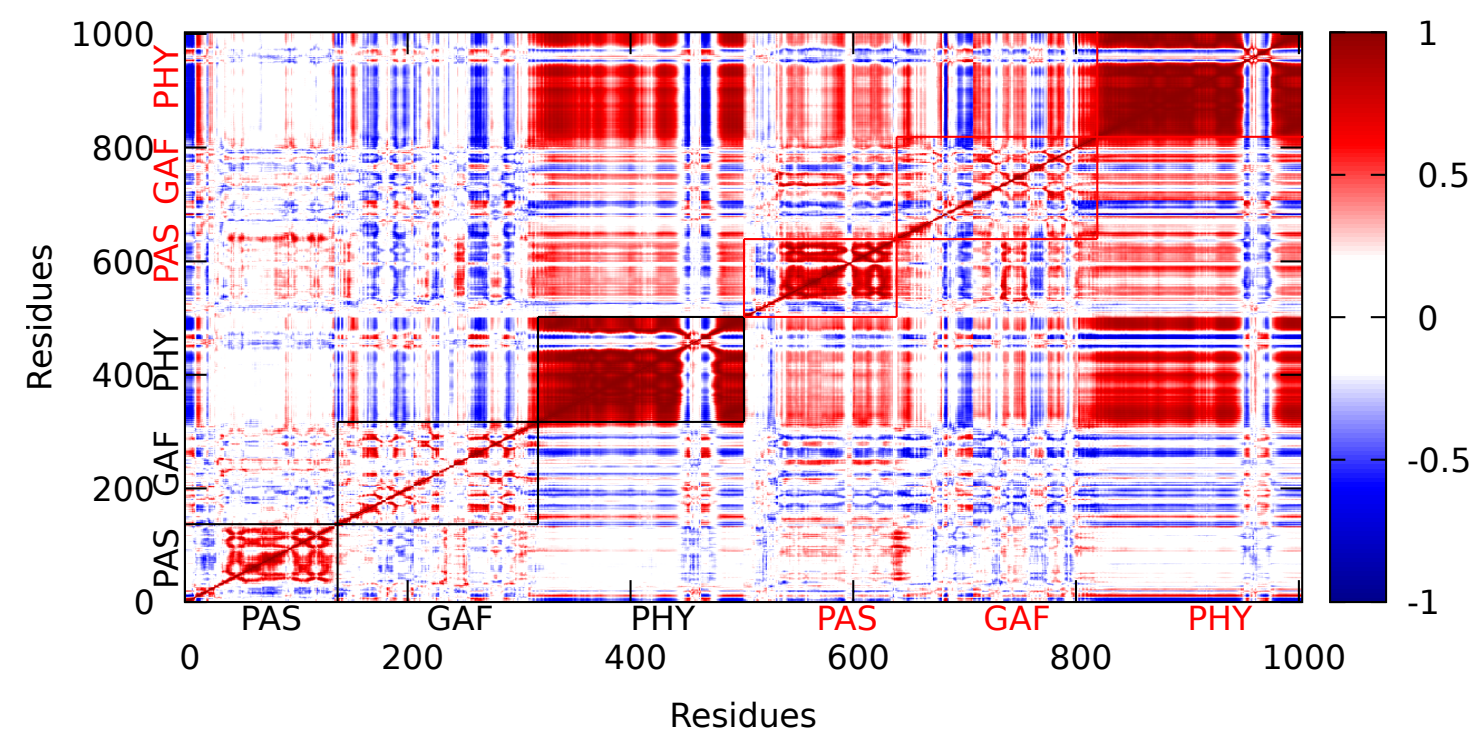

Figure S36: DCC along the $\mathrm{z}$ direction for the DrBphP dimer from aMD with CHARMM 27

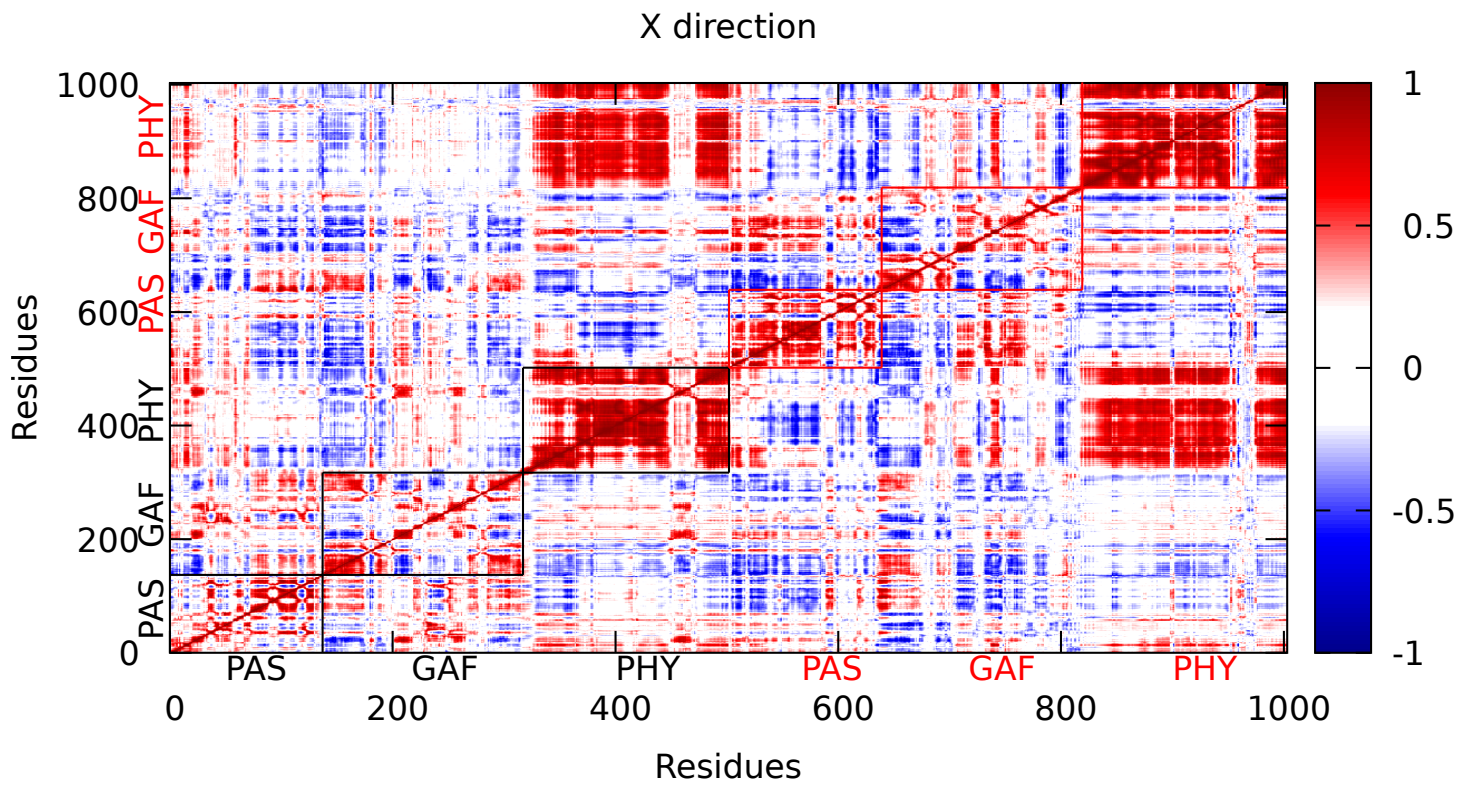

Figure S37: DCC along the $\mathrm{x}$ direction for the DrBphP dimer from GaMD with AMBER ff14SB. 
Y direction

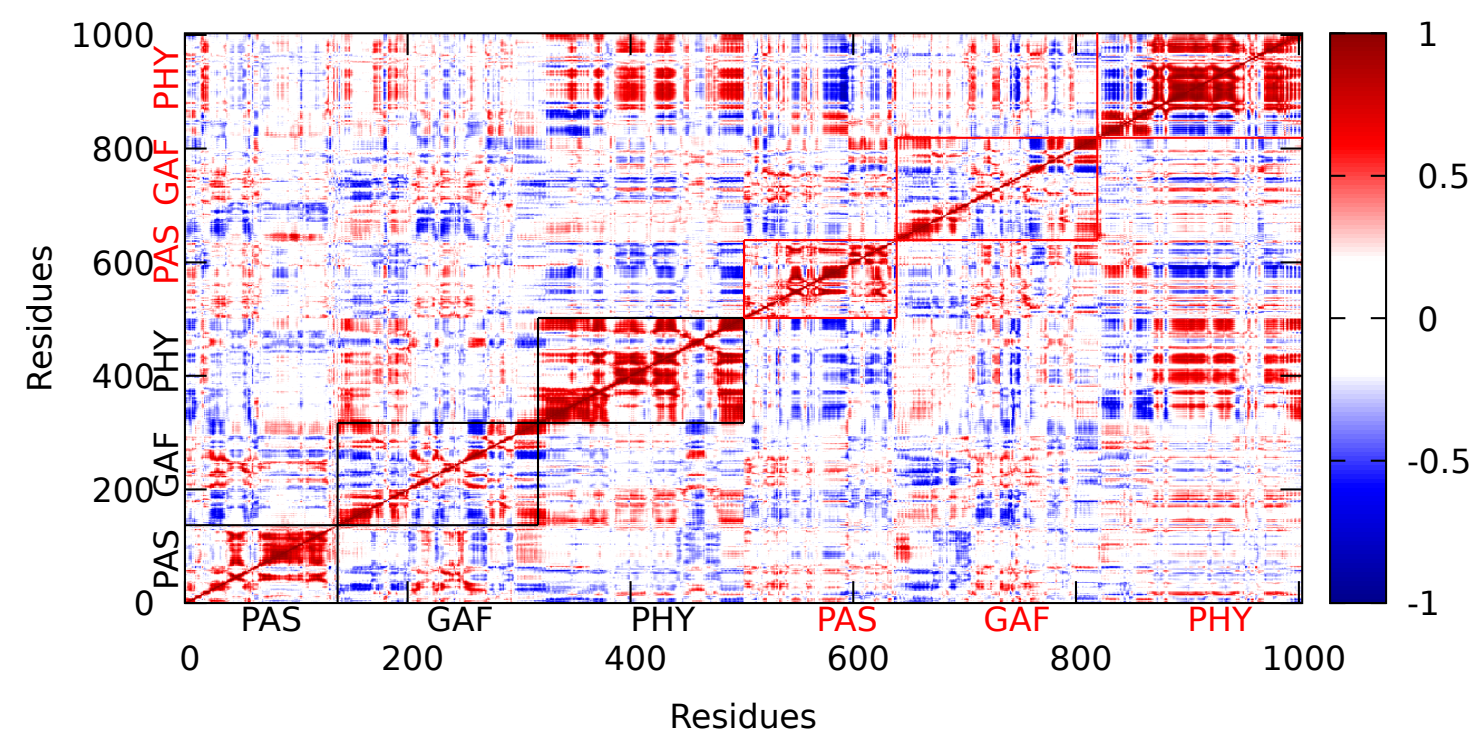

Figure S38: DCC along the y direction for the DrBphP dimer from GaMD with AMBER ff14SB.

\section{$Z$ direction}

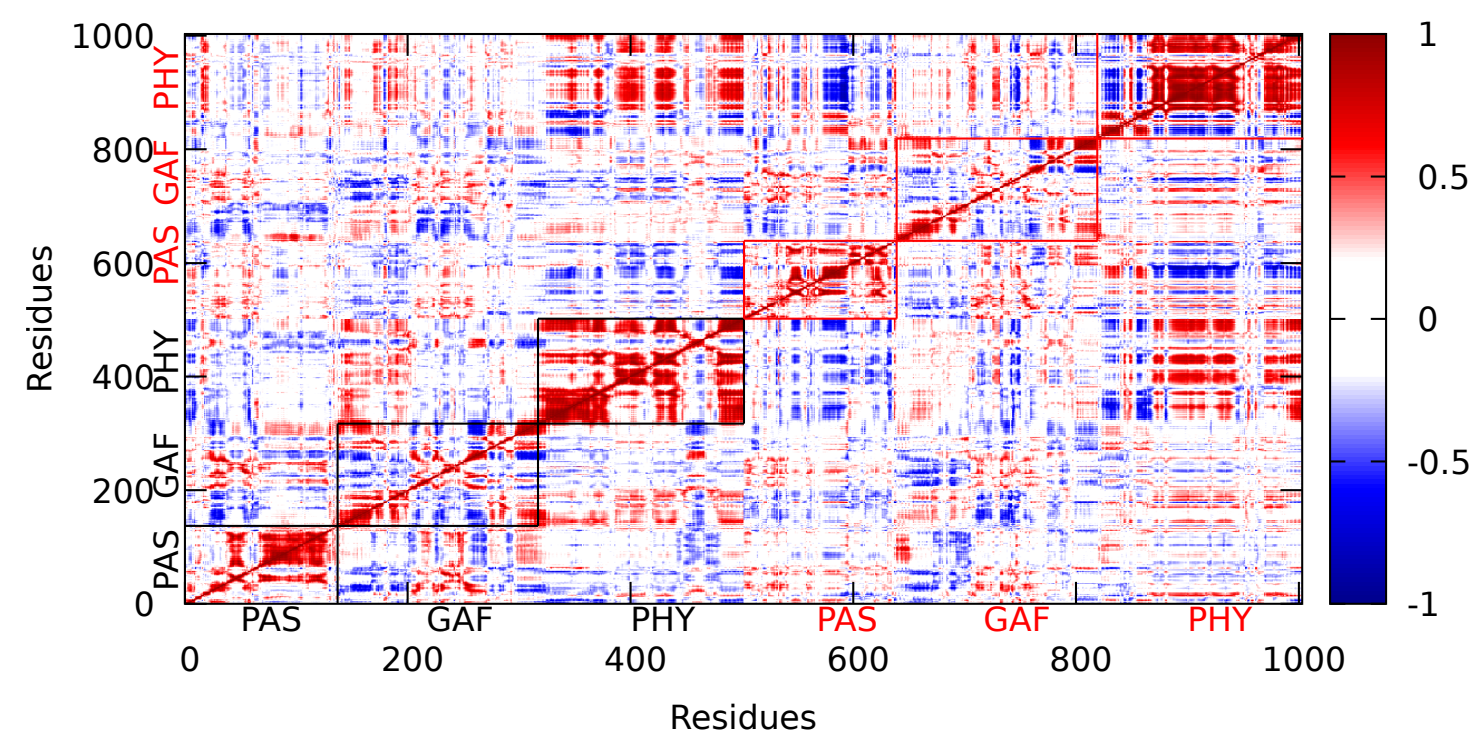

Figure S39: DCC along the $\mathrm{z}$ direction for the DrBphP dimer from GaMD with AMBER ff14SB. 


\section{References}

[1] Steve Kaminski, Grazia Daminelli, and Maria Andrea Mroginski. Molecular dynamics simulations of the chromophore binding site of deinococcus radiodurans bacteriophytochrome using new force field parameters for the phytochromobilin chromophore. The Journal of Physical Chemistry B, 113(4):945-958, 2009. PMID: 19123828.

[2] Olgun Guvench and Alexander D. MacKerell. Automated conformational energy fitting for force-field development. Journal of Molecular Modeling, 14(8):667-679, Aug 2008.

[3] Chad W. Hopkins and Adrian E. Roitberg. Fitting of dihedral terms in classical force fields as an analytic linear least-squares problem. Journal of Chemical Information and Modeling, 54(7):1978-1986, 2014. PMID: 24960267.

[4] James A. Maier, Carmenza Martinez, Koushik Kasavajhala, Lauren Wickstrom, Kevin E. Hauser, and Carlos Simmerling. ff14sb: Improving the accuracy of protein side chain and backbone parameters from ff99sb. Journal of Chemical Theory and Computation, 11(8):3696-3713, Aug 2015.

[5] Karl T. Debiec, David S. Cerutti, Lewis R. Baker, Angela M. Gronenborn, David A. Case, and Lillian T. Chong. Further along the road less traveled: Amber ff15ipq, an original protein force field built on a self-consistent physical model. Journal of Chemical Theory and Computation, 12(8):3926-3947, Aug 2016.

[6] David S. Cerutti, William C. Swope, Julia E. Rice, and David A. Case. ff14ipq: A self-consistent force field for condensed-phase simulations of proteins. Journal of Chemical Theory and Computation, 10(10):4515-4534, Oct 2014. 